OPEN ACCESS

Edited by:

Ming Yann Lim,

Tan Tock Seng Hospital, Singapore

Reviewed by:

Aristeidis G. Telonis,

University of Miami, United States

Xiaobai Liu,

ShengJing Hospital of China Medical

University, China

*Correspondence:

Chun Luo

luochuntongji@sina.com

Qi Wang

dr_wang_qi@outlook.com

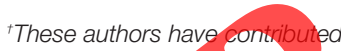

equally to this work

Specialty section:

This article was submitted to

Head and Neck Cancer,

a section of the journal

Frontiers in Oncology

Received: 30 August 2020 Accepted: 30 March 2021

Published: 23 April 2021

Citation:

Cui Y, Wang Q, Lin J, Zhang L, Zhang C, Chen H, Qian J and Luo C (2021) miRNA-193a-3p Regulates

the AKT2 Pathway to Inhibit the

Growth and Promote the Apoptosis of Glioma Cells by Targeting ALKBH5.

Front. Oncol. 11:600451.

doi: 10.3389/fonc.2021.600451

\section{miRNA-193a-3p Regulates the AKT2 Pathway to Inhibit the Growth and Promote the Apoptosis of Glioma Cells by Targeting ALKBH5}

\author{
Yong Cui ${ }^{1,2 t}$, Qi Wang ${ }^{2 \star t}$, Jing Lin $^{3}$, Lei Zhang ${ }^{2}$, Chi Zhang ${ }^{2}$, Huairui Chen ${ }^{2}$, Jun Qian ${ }^{2}$ \\ and Chun $\mathrm{Luo}^{2 *}$ \\ ${ }^{1}$ Department of Neurosurgery, Third Affiliated Hospital of Naval Medical Univer \\ 2 Department of Neurosurgery, Shanghai Tongji Hospital, Tongji University Sch \\ ${ }^{3}$ Department of Neurosurgery, Changzheng Hospital of Naval Medical University,
}

Emerging evidence indicates that microRNA (miR)-193a-3p is involved in the tumor progression of various cancers. However, the biological functions and precise molecular mechanisms of miR-193a-3p in gliömas have not been well documented. Accordingly, this study focused on the tumor suppressor role and molecular mechanisms of miR-193a-3p in glioma cells. miR-193a-3p expression was determined by qRT-PCR in glioma tissues and cell lines 1251 and U87 glioma cells were transfected with a miR193a-3p mimic. The effects of miR-193a-3p on cell growth and apoptosis were investigated using MIT, colony-forming, and flow cytometry assays. Overexpression of miR-1932-3p in U87 cells also significantly suppressed tumorigenicity and induced apoptosis in the xenograft mouse model. Luciferase assays were conducted to determine if ALKBH5 is a direct target of miR-193a-3p in glioma cells. Inmenopreeipitation was used to explore the interaction between ALKBH5 and RACserine/threonine-protein kinase 2 (AKT2) in glioma cells. miR-193a-3p was downregulated in glioma tissues and cell lines. miR-193a-3p treatment suppressed proliferation and promoted apoptosis in both U251 and U87 cells. Bioinformatics analysis and luciferase reporter assay identified a novel miR-193a-3p target, ALKBH5. Notably, the antitumor effect of miR-193a-3p transfection in glioma cells may be due to the miR-193a-3p-induced inhibition of AKT2 expression caused by the suppression of ALKBH5 expression. Furthermore, immunoprecipitation indicated that ALKBH5 physically interacted with AKT2 through an RNA-independent mechanism in glioma cells. miR-193a-3p directly targets ALKBH5 to inhibit the growth and promote the apoptosis of glioma cells by suppressing the AKT2 pathway both in vitro and in vivo, and the physical interaction between ALKBH5 and AKT2 is essential for suppressing cell apoptosis by upregulating miR-193a-3p in glioma cells. Our study revealed that the antitumor effects of miR-193a-3p on glioma cells is due to ALKBH5 mediation of the AKT2-induced intrinsic apoptosis signaling pathway.

Keywords: glioma, miR-193a-3p, proliferation, apoptosis, ALKBH5 


\section{INTRODUCTION}

Gliomas, which originate from glial cells, represent the most frequent primary malignant tumors of the central nervous system in humans (1). Glioblastoma is the most aggressive form of human astrocytoma with a median survival of only 14-15 months (2). The main cause of mortality in patients with glioma is the apoptosis resistance of individual tumor cells (3). Therefore, it is an urgent need to understand the molecular mechanisms underlying the tumorigenesis of glioma, thus contributing to figure out novel molecular markers to achieve new treatment possibilities.

MicroRNAs (miRNAs), a group of single-stranded noncoding RNAs, may serve as oncogenes or tumor suppressors by degrading target mRNA in gliomagenesis $(4,5)$. miR-193a-3p, located in the human 17q11.2 gene locus, has been reported to play an important role in the physiological and pathological processes involved in cell proliferation, angiogenesis, and apoptosis (6-8). Cells of different type of malignant tumors, such as colon, prostate, lung, and breast cancers show the low expression of miR-193a-3p, suggesting miR-193a-3p is a tumor suppressor with inhibiting activity towards tumor development (8-10). For instance, miR-193a-3p inhibits ovarian cancer growth, migration and angiogenesis by directly targeting growth factor receptor-bound protein-7 (GRB7) (11). There was a report that GRB7 knockdown impairs glioma growth and associated angiogenesis in vivo (12). Notably, Kwon et al. (6) revealed that miR-193a-3p was increased in U251 glioma cells treated with ionizing radiation and that miR-193a-3p may be an important mediator of the accumulation of intracellular reactive oxygen-species and DNA damage. However, its clinical significance, expression pattern, and biological functions in glioma are still unclear.

ALKBH5, also known as alkB homologue 5, is a member of the specific mRNA ${ }^{6} \mathrm{~A}$ demethylase family. ${ }^{6} \mathrm{~A}$ is methylated during nascent pre-mRNA processing by a methyltransferase complex and is demethylated by fat mass and obesity-associated protein (FTO) and ALKBH5 $(13,14)$, Emerging evidence demonstrate that methyltransferase serves as a multifunctional adaptor that contributes to the regulation of cancer cell progression via noncoding RNAs (ncRNAs), such as miRNAs and long non-coding RNAs (lncRNAs) $(15,16)$. For instance, $\mathrm{m}^{6} \mathrm{~A}$ modification influences arsenite-induced carcinogenesis by modifying various miRNAs (miR-106b, miR-18a/b, miR-3607, miR-423, miR-30a, miR-320b/d/e) (17). Specifically, it was reported that an IncRNA antisense to forkhead box M1 (FOXM1-AS) promotes the interaction of ALKBH5 with FOXM1 transcripts to increase glioblastoma stem-like cells tumorigenesis (18). Thus, it is reasonable to speculate that the interplay between ALKBH5 and ncRNAs may play an important role in contributing to glioma progression. Recently, a positive feedback regulation between miR-193a-3p and ALKBH5 has been identified in esophageal squamous cell carcinoma (19). However, the precise molecular mechanisms underlying the miR-193a-3p-mediated ALKBH5 signaling pathway remain largely unknown.
Here, we confirmed that miR-193a-3p was significantly underexpressed in glioma specimens. Overexpression of miR193a-3p in U251 and U87 glioma cells inhibited cell growth and enhanced cell apoptosis. In addition, ALKBH5 was identified as a direct target of miR-193a-3p, and it was clarified that the miR193a-3p-ALKBH5-RAC-serine/threonine-protein kinase 2 (AKT2) signals leads to the mediation of tumor growth and cell apoptosis of U87 and U251 cells. Furthermore, we demonstrated that ALKBH5 can physically interact with AKT2 through an RNA-independent mechanism in glioma cells.

\section{METHODS}

\section{Tissue Samples}

Glioma tissues were collected from patients with glioma at PLA 411 Hospital from January 2010 to December 2016. The use of glioma tissue was approved by the Specialty Committee on Ethics of Biomedicine Research (PJ2010-012-03) of PLA 411 Hospital. Patients receiving chemotherapy or radiotherapy prior to the surgery were excluded. The control bram tissue samples were from the adjacent normal brain tissues of traumatic brain injury patients. The clinicopathologic characteristics of the patients are summarized in Table 1. A detailed table with the patient's characteristics is described in Table S1.

\section{miRNAs and Transfection}

Synthetic pre-miR-193a-3p, anti-miR-193a-3p, pre-miRcontrol (miR-NC), and anti-miR-control RNAs (anti-NC) were purchased from GenePharma (Shanghai, China). All cells were seeded at $1 \times 10^{6}$ cells per plate overnight until $50 \%$ confluent and then non-targeting controls (miR-NC), miR-193a-3p inhibitor, and miR-193a-3p mimic were added. Cells were transfected with $100 \mathrm{nM}$ miR-193a-3p mimic, NC mimic, anti-miR-193a-3p or anti-NC, using Lipofectamine ${ }^{\circledR}$ RNAiMAX Transfection Reagent (Invitrogen) according to the manufacturer's protocol. Cells were incubated at $37^{\circ} \mathrm{C}$ in $5 \% \mathrm{CO}_{2}$ for $24 \mathrm{~h}$ with antibiotic-free medium. Complete medium containing the mixture from the above cultures was then added to the plates after removal of the old medium. A short hairpin

TABLE 1 | Clinicopathologic characteristics of 63 patients with glioblastomas.

\begin{tabular}{lc} 
Characteristic & n (\%) \\
\hline $\begin{array}{l}\text { Age(y) } \\
<=50\end{array}$ & $25(39.68)$ \\
$>50$ & $38(60.32)$ \\
Gender & \\
$\quad$ Male & $41(65.09)$ \\
Female & $22(34.91)$ \\
Size of tumor & \\
$\quad<=4 c m$ & $27(42.86)$ \\
$>4 c m$ & $36(57.14)$ \\
Radiotherapy & $54(85.71)$ \\
Chemotherapy & $52(82.54)$ \\
Patient survival & \\
$\quad$ Alive & $17(26.98)$ \\
Deceased & $46(73.02)$
\end{tabular}


sequence targeting ALKBH5 (5'-CCGGGGAAAG GCTGTT GGCA TCAATACTCG AGTATTGATG CCAACAGCCT TTCTTTTTG-3') (Invitrogen, Carlsbad, CA) and a scrambled sequence (5'-CCGGGGAAAG GATGTAAAGA AGTATG TATT TCAAGAGAAT ACATACTTCT TTCGAGTATT ATTTTTGGAA AT-3') was inserted into pLVTHM vector. Full-length human ALKBH5 complementary DNA tagged with GFP (Lenti-ALKBH5) or GFP alone (Lenti-GFP) was cloned into the LENTI-BISVECTOR (Invitrogen).

\section{Cell Proliferation Assay}

3-(4,5-Dimethyl-2-thiazolyl)-2,5-diphenyl-2-H-tetrazolium bromide (MTT) assay was used to assess cell viability. Cells were seeded in 96-well plates $24 \mathrm{~h}$ after transfection and treated with $0.2 \mathrm{mg} / \mathrm{ml} \mathrm{MTT}$ for $4 \mathrm{~h}$ at $37^{\circ} \mathrm{C}$. The absorbance was read at 570 $\mathrm{nm}$. The optical density at $570 \mathrm{~nm}$ was measured $24,48,72,96$ and $120 \mathrm{~h}$ after transfection.

\section{Western Blot Analysis}

Western blot analysis was conducted as previously described (20). The primary antibodies were ALKBH5 (ab-195377, 1:1,000; Abcam, Cambridge, MA), AKT2 (, CST-L79B2, 1:600; Cell Signaling Technology, Danvers, MA), B cell lymphoma (Bcl)-2 (abcam-185002, 1:1000; Abcam), baculoviral inhibitor of apoptosis repeat-containing 5 (Survivin; sc-101433, 1:500; Santa Cruz Biotechnology, Dallas, TX), Bcl-2-associated X protein (Bax; sc-7480, 1:500; Santa Cruz Biotechnology), Bcl-2-associated death promoter (Bad; sc-8044, 1:500; Santa Cruz Biotechnology), glyceraldehyde 3-phosphate dehydrogenase (GAPDH; ab-110305, 1:800; Abcam), and $\beta$-actin (ab-8227, $1: 1,000$; Abcam). $\beta$-actin was utilized for normalization-of the signal intensity of each protein.

\section{Colony Formation Assay}

Cells were cultured in DMEM at $37^{\circ} \mathrm{C}$ in $5 \% \mathrm{CO}_{2}$. After 14 days, the cells were washed twice with PBS and stained with Griemsa solution. The number of colonies containing $>50$ cells was determined. Cell viability was analyzed with a Cell Counting Kit (cat. no. 11465007001, Roche Diagnostics GmbH). The optical density at $570 \mathrm{~nm}$ was measured 24, 48, 72, 96 and $120 \mathrm{~h}$ after transfec

\section{Luciferase Reporter Assay}

U87 cells were seeded in 24-well plates and incubated for $24 \mathrm{~h}$ before transfection. The cells were transfected with ALKBH5-3' UTR-WT (wild-type) or ALKBH5-3'UTR-MT (mutant) with miR-193a-3p mimic or miR-NC. The Dual-Luciferase Reporter Assay System (Promega, Madison, WI) was used to measure the luciferase activity after transfection at $24 \mathrm{~h}$.

\section{Quantitative RT-PCR Analysis}

Tumor specimens were frozen at $-75^{\circ} \mathrm{C}$ before experiments. For miR-193a-3p analysis, the TaqMan MicroRNA Reverse Transcription Kit was used to conduct reverse transcription (Applied Biosystems, Foster City, CA). qRT-PCR was conducted by using the Platinum SYBR Green qPCR SuperMix-UDG (Qiagen, Germantown, MD) for mRNA analysis. The selective prime sets obtained from Exiqon included hsa-miR-193a-3p (Product No. 204591) and hsa-miR193b-3p (Product No. 204226), as described by previous study (11). The forward and reverse primers were as follows: ALKBH5 (forward) 5'-CACATCCTGG AAGGCAGCAA-3' and (reverse) 5'-CCCCCAAAGT GGTGGTATCC-3'; and $\beta$-actin (forward) 5'-ACCAACTGGG ACGACATGGA GAAA-3' and (reverse) 5'TAGCACAGCC TGGATAGCAA CGTA-3'. The thermocycling parameters were as follows: $95^{\circ} \mathrm{C}$ for $10 \mathrm{~min}$, followed by 40 cycles at $95^{\circ} \mathrm{C}$ for $15 \mathrm{sec}, 60^{\circ} \mathrm{C}$ for $1 \mathrm{~min}$, and a detection step at $72^{\circ} \mathrm{C}$ for $30 \mathrm{sec}$.

\section{Immunoprecipitation}

The cells were washed twice with PBS and linked with 12000 $\mathrm{J} / \mathrm{cm}^{2} \mathrm{UV}$ light for $2 \mathrm{~min}$. The lysate is treated with $30 \%$ amplitude ultrasound for 5 minutes, 3 seconds pulse, followed by 6 seconds rest period. 50 $\mu$ l Dynabeads Protein G magnetic

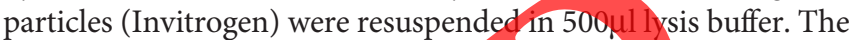
beads were precipitated by magnet and then suspended again in the clear lysate and incubated at $4^{\circ} \mathrm{C}$ for $2 \mathrm{~h}$. All beads were resuspended in lysis buffer and subjected to western blots.

\section{Immunohistochemistry (IHC) and Expression Analysis}

Three-micrometer-thick, paraffn-embedded, human tissue sections were deparaffinized in xylol and rehydrated in a graded ethanol series. Antigen retrieval was performed by microwave heating for $20 \mathrm{~min}$ in $1 \mathrm{mM}$ EDTA buffer ( $\mathrm{pH} 8.0$ ). The sections were incubated in nonimmune serum for $30 \mathrm{~min}$ and then incubated overnight at $4^{\circ} \mathrm{C}$ in ALKBH5 antibody (ab195377, 1:500; Abcam) or Ki-67 antibody (9449S, 1:500, CST). Immunostaining was classified according to the percentage of positive cells and to the staining intensity. Scores for staining intensity were assigned as follows: 0 for no staining, 1 for light brown, 2 for brown, and 3 for dark brown. Scores for the percentage of positive cells were assigned as follows: 0 for $<10 \%$ cells positive; 1 for $11 \%$ - $25 \%$ cells positive; score 2 for $26 \%$ $50 \%$ cells positive; score 3 for $51 \%-75 \%$ cells positive; and score 4 for $>75 \%$ cells positive. Overall scores were obtained by multiplying the percent positive score by the intensity score. Two independent pathologists examined five random fields ( 1 field $=0.159 \mathrm{~mm}^{2}$ at $\times 100$ magnification ) in each sample.

\section{MeRIP-qPCR}

M6A modifications of individual genes were measured by the Magna MeRIP Kit. Briefly, $5 \mu \mathrm{g}$ anti-m6A antibody (202,003, Synaptic Systems) or rabbit IgG was added to a tube containing Pierce $^{\text {TM }}$ Protein A/G Magnetic Beads $(88,803$, Thermo Scientific), followed by rotation at RT for $30 \mathrm{~min}$. Then, the methylated mRNAs were stored at $-80^{\circ} \mathrm{C}$ overnight. The beads were resuspended in $900 \mu \mathrm{L}$ RIP immunoprecipitation buffer mixed with $100 \mu \mathrm{L}$ cell lysate and extracted with RIP wash buffer. Further enrichment was calculated by qPCR.

\section{Apoptosis Assay}

ApoScreen Annexin V Apoptosis Kit (Bender MedSystem, Burlingame, CA) was used to measure cell apoptosis. After being 
washed twice with PBS, cell samples were collected and FITC was added. Flow cytometry (FACScan; BD Biosciences, San Jose, CA) was conducted to analysis all cells. Data analyses were performed using CellQuest software Version 5.0 (BD Biosciences). Experiments were performed three times.

\section{Intraperitoneal Human Glioblastoma Xenograft Model}

For the study, 3-week-old BABL/c female nude mice were used and randomized into two groups: miR-NC (negative control) and miR-193a-3p mimics. Approximately $2 \times 10^{6}$ human U87 glioma cells were inoculated subcutaneously of nude mice as described previously (21). 31 days post-injection, mice were sacrificed before separating the tumors and weighting them. Formalin-fixed tumors were analyzed for proliferation using BrdUrd incorporation. Purified tumors were evaluated for apoptosis by TUNEL of fragmented DNA. All animal experiments were approved by the Animal Care Committee of PLA 411 hospital.

\section{Statistical Analysis}

Statistical analysis was performed using the Student's t-test. Comparisons between three or more groups were performed using analysis of variance (ANOVA), followed by Dunnett's ttest. The Kaplan-Meier (K-M) method for univariate survival analysis was conducted to analysis survival differences between groups. Spearman's correlation coefficient was used to investigate the correlation between miR-193a-3p and ALKBH5 expression. Multivariate survival analysis using the Cox proportional hazards model was performed to assess predictors related to survival. Differences were considered statistically significant when $\mathrm{P}<0.05$.

\section{RESULTS

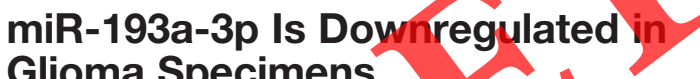 \\ Glioma Specimens}

We first investigated the expression levels of miR-193a-3p in 78 human glioma specimens and 32 normal brain tissues by qRTPCR. The expression level of miR-193a-3p was significantly lower in glioma tissues compared with the control normal tissues (Figure 1A). We next examined the expression of miR193a-3p in low-grade (IDH wild-type, diffuse astrocytoma; $\mathrm{n}=28$ ) and high-grade (IDH wild-type, glioblastoma; $n=63$ ) tumor specimens. As illustrated in Figure 1B, miR-193a-3p expression was significantly decreased in high-grade glioma tissues (glioblastomas). The same 63 glioblastomas were divided into the low $(<3.45 ; \mathrm{n}=39)$ and high $(\geq 3.45 ; \mathrm{n}=24)$ miR-193a-3p group using qRT-PCR analysis and subsequently subjected to assess the association between miR-193a-3p expression and clinicopathologic features (Table 1). Ki-67, a critical cellular marker for proliferation, expression was significantly lower in miR-193a-3p high glioblastoma (high grade) than that in miR-193a-3p low group $(\mathrm{P}<0.05$; Figure 1C) as measured by IHC. Spearman's correlation analysis further revealed that the miR-193a-3p expression was inversely correlated with Ki-67 expression in patients with glioblastomas (high-grade) $(\mathrm{P}<0.01$; Figure 1D), while no significant correlation was observed between miR-193a-3p and Ki-67 expression in patients with low-grade gliomas $(\mathrm{P}=0.07$; Figure 1E). Collectively, miR-193a-3p expression is reversely associated with the proliferative activity of high-grade gliomas and with tumor progression.

Since diffuse astrocytomas (low-grade) are considered benign with long-term survival (22), we did Kaplan Meier plots of miR193a-3p high/low and overall survival rate for each grade gliomas separately (diffuse astrocytomas-low grade, $\mathrm{n}=28$, and glioblastomas-high grade, $\mathrm{n}=63$ ) to make survival analysis more convincing. As shown by K-M survival curves, survival probability was promoted in patients with miR-193a-3p-high glioblastomas $(\mathrm{P}=0.0277$; Figure 1F). In low grade gliomas, the expression of miR-193a-3p was not correlated with survival $(\mathrm{P}=0.1$; Figure 1G). Univariate survival analysis revealed that miR-193a-3p expression and age at diagnosis were significantly associated with prognosis, whereas no significant associations were indicated between prognosis and other clinicopathologic features (gender, tumor size, chemotherapy, or radiotherapy) (Table 2). Multivariate analysis revealed that miR-193a-3p expression was a significant prognostic factor for patients with glioblastoma (Table 2). All these results indicated that miR193a-3 might be a key prognostic index for glioblastoma (highgrade) patient survival.

miR-193a-3p Overexpression Suppresses the Growth and Enhances the Apoptosis of Glioma Cells

We first measured the miR-193a-3p expression in five glioma cell lines, including A172, SGH44, LN229, U251, and U87, by qRTPCR analysis (Figure 2A). U251 and U87 malignant glioma cells had significantly lower expression of miR-193a-3p compared with that of A172, SGH44, and LN229 cells. Therefore, U251 and U87 cells were used to produce the next experiments. To explore the antitumor effects of miR-193a-3p in glioma cells, we transfected glioma cell lines with miR-193a-3p mimics to upregulate miR-193a-3p expression. RT-PCR showed that miR-193a-3p mimics clearly increased miR-193a-3p expression in U251 and U87 cells, whereas no changes were observed in miR-193b-3p expression in glioma cells (Figures 2B and S1A). These results confirmed that miR-193a-3p is actually influenced by miR-193a-3p mimics without affecting miR-193b-3p expression in glioma. Consistent with a critical role of miR193a-3p in cell survival, miR-193a-3p mimic-transfected U251 and U87 cells had significantly lower cell growth than miR-NC, as measured by MTT assay (Figure 2C). Colony-forming assays showed that upregulation of miR-193a-3p in glioma cell lines inhibited anchorage-independent growth (Figure 2D). In addition, overexpression of miR-193a-3p resulted in a significant increase in glioma cell apoptosis (Figure 2E). Moreover, transmission electron microscope analysis was performed to confirm the inducing apoptosis of miR-193a-3p on glioma cells. It is shown that several classic apoptotic changes 
A

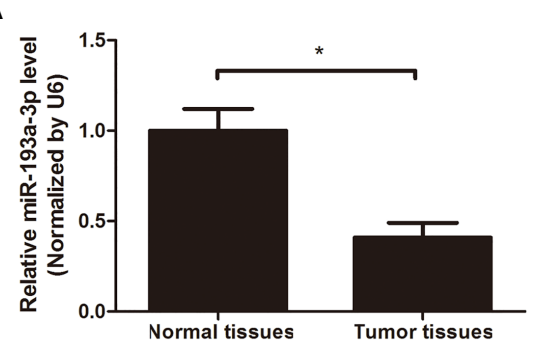

B

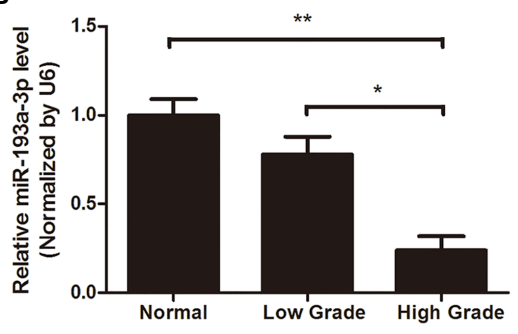

C

miR-193a-3p High
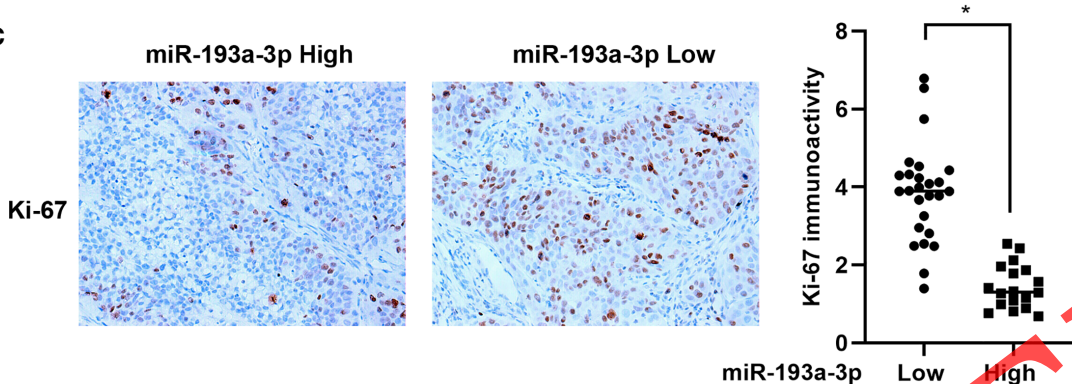

D

High Gade

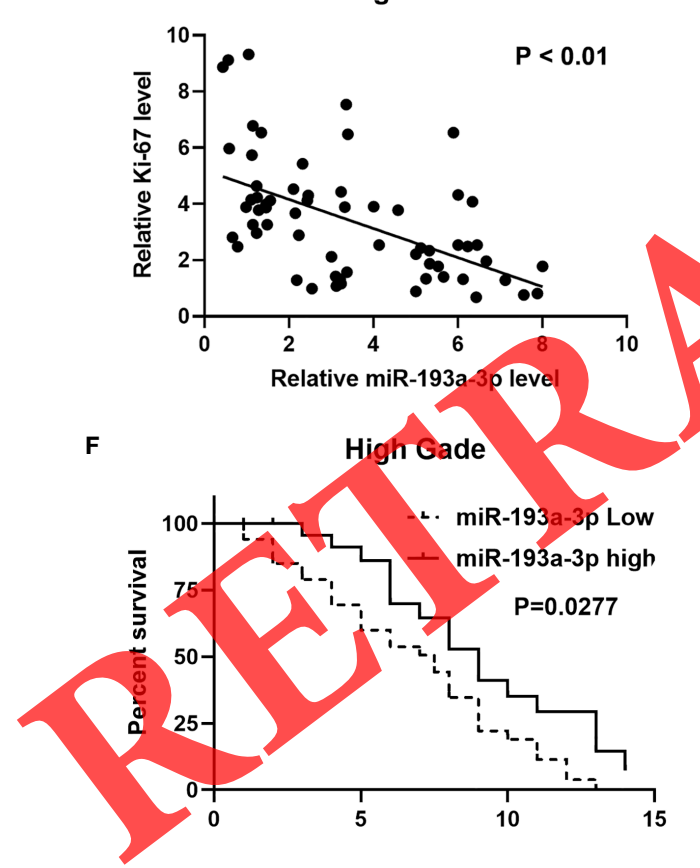

$\mathbf{F}$

High Gade

E

miR-193a-3p Low high

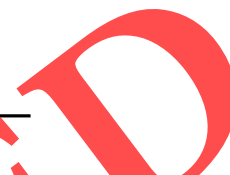

rade

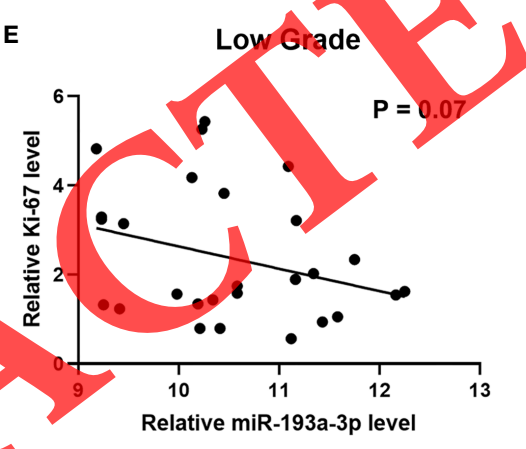

Low Grade

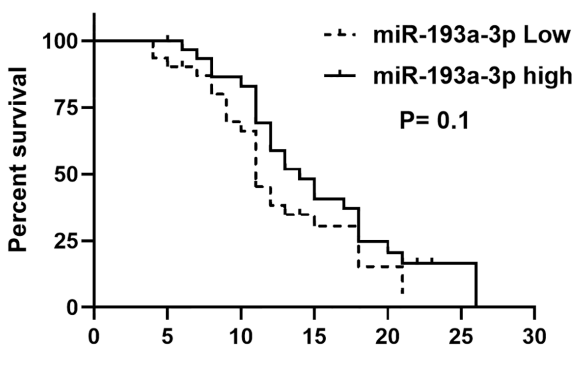

FIGURE 1 | (A) Expression levels of miR-193a-3p in glioma tissues (tumor tissues) and the adjacent tissues (normal tissues) were determined by qRT-PCR. ${ }^{*} P<0.05$. (B) Expression levels of miR-193a-3p in gliomas of different pathological grade were determined by $q R T-P C R$. ${ }^{*} P<0.05$ and ${ }^{* *} P<0.01$. (C). Left: Representative immunostaining showed expression of Ki-67 in miR-193a-3p high and in miR-193a-3p low-expressing tumors. Right: Quantitative analysis of Ki-67 immunoactivity. ${ }^{*} \mathrm{P}<0.05$. (D) Correlation between Ki-67 inmmunocativity and miR-193a-3p expression in high-grade gliomas. $r=-0.53, P<0.01$. (E) No significant correlation was found between Ki-67 inmmunocativity and miR-193a-3p expression in low-grade gliomas. $r=-0.33, P=0.07$. (F) Kaplane-Meier survival curves in patients with high-grade gliomas according to the expression of miR-193a-3p. Patient tumors were analyzed by qRT-PCR for miR-193a-3p expression and divided into high and low expression groups as described in Results. $P=0.00277$. (G) No significant correlation was found between miR-193a-3p expression and survival probability in low-grade gliomas. $\mathrm{P}=0.01$.

were found in glioma cells underexpressing miR-193a-3p, such as nuclear condensation and fragmentation and cell blebbing morphological alterations (Figure 2F). These data strongly suggest that miR-193a-3p can inhibit glioma cell survival by promoting cell apoptosis.

\section{miR-193a-3p-Induced Apoptosis Is Correlated With Suppression of AKT2}

Previously, we found that the AKT2 signaling pathway participate in regulating glioma cellular growth and apoptosis (21). To figure out whether AKT2 pathway activation contributes 
TABLE 2 | Univariate and multivariate analysis of different prognostic parameters in patients with glioblastomas by Cox regression analysis.

\begin{tabular}{|c|c|c|c|c|}
\hline Variable & No.patient & $\begin{array}{l}\text { univariate log } \\
\text {-rank test(p) }\end{array}$ & $\begin{array}{c}\text { Cox multivariable } \\
\text { analysis(p) }\end{array}$ & $\begin{array}{c}\text { relative } \\
\text { risk }\end{array}$ \\
\hline \multicolumn{5}{|l|}{ Age(y) } \\
\hline$<=50$ & 25 & 0.023 & 0.041 & 5.372 \\
\hline$>50$ & 38 & & & \\
\hline \multicolumn{5}{|l|}{ Gender } \\
\hline Male & 41 & 0.912 & 0.675 & NS \\
\hline Female & 22 & & & \\
\hline \multicolumn{5}{|l|}{ Size of tumor } \\
\hline$<=4 \mathrm{~cm}$ & 27 & 0.792 & 0.618 & NS \\
\hline$>4 \mathrm{~cm}$ & 36 & & & \\
\hline Radiotherapy & 54 & 0.179 & 0.112 & NS \\
\hline Chemotherapy & 52 & 0.431 & 0.396 & NS \\
\hline \multicolumn{5}{|l|}{ miR-193a-3p } \\
\hline low & 39 & 0.012 & 0.031 & 5.958 \\
\hline high & 24 & & & \\
\hline
\end{tabular}

NS, No significant.

to the miR-193a-3p-induced apoptosis, we examined the expression and activations of AKT signaling pathway molecules and cellular apoptosis pathways. As illustrated in Figure 2G, the expression levels of both total AKT2 and phosphorylated AKT2 (p-AKT2) were significantly decreased in U251 and U87 cells transfected with miR-193a-3p mimics, whereas the levels of total AKT1, total AKT3, p-AKT1, and pAKT3 did not appear to differ between the two groups. Furthermore, we found upregulation of miR-193a-3p resulted in a significant decrease in the expression levels of the antiapoptotic proteins Bcl-2 and Survivin, while no changes in the levels of the apoptotic proteins Bax and Bad were visualized after miR-193a-3p transfection (Figure 2G). These data strongly indicate that miR-193a-3p may suppress the activity of the AKT2 signaling pathway in glioma cells, which uitimately promotes glioma cell apoptosis by suppressing the levels of the important antiapoptotic proteins Bcl-2 and Survivin.

\section{ALKBH5 Is a Novel Target of miR-193a-3p}

To further explore the molecular mechanism on $m i R-193 a-$ $3 p$-mediated proliferation of gliomas, we used databases of miRmap (https://mirmap.ezlab.org/app/), TargetScan (http:// www.targetscan.org/vert 72/) and RNA22 (https://cm.jefferson. edu/rna22/) to search for target genes that have an established function in regulating glioma cell growth (Figure S1B and Table S2). Among the candidate potential target genes, we found that ALKBH5 has a miR-193a-3p binding site in its 3'UTR (Figure 3A). We then used a luciferase reporter gene assay to validate whether miR-193a-3p regulates ALKBH5 expression. As measured by luciferase reporter gene assay, overexpression of miR-193a-3p in U87 cells led to a dramatic decrease in the luciferase activity of the ALKBH5 WT $3^{\prime}$-UTR (Figure 3B). In addition, qRT-PCR and western blot analysis showed that miR-193a-3p mimics transfection resulted in substantially inhibiting endogenous ALKBH5 mRNA and protein levels in U87 cells (Figures 3C, D). Unlike U87 cells that transfected with miR-193a-3p mimics, U87 cells treated with miR-193a-3p inhibitor showed higher ALKBH5 mRNA and protein expression levels compared to the control (Figures 3C, D). In addition, we investigated the association between the miR193a-3p level and ALKBH5 expression in glioma specimens to identify that ALKBH5 was a direct target of miR-193a-3p. IHC showed that ALKBH5 expression was significantly lower in miR193a-3p high-expressing group compared with that in miR-193a$3 p$ low-expressing group $(\mathrm{P}<0.05$; Figure $3 \mathrm{E})$. qRT-PCR analysis revealed the similar results at mRNA expression levels, which is consistent with the IHC results ( $\mathrm{P}<0.05$; Figure $3 \mathrm{~F})$. Spearman's correlation analysis confirmed the results that the miR-193a-3p expression was decreased and inversely correlated with elevated level of ALKBH5 in patients with gliomas. ( $\mathrm{r}=-0.503, \mathrm{P}<0.01$; Figure 3G). All these data indicate that miR-193a-3p reduces ALKBH5 expression though its $3^{\prime}$-UTR and that ALKBH5 is a novel downstream target of miR-193a-3p in gliomas.

\section{ALKBH5 Functions as an Oncogene in Gliomas}

We examined ALKBH5 mRNA and protein expression levels in A172, SGH44, LN229, U251, and U87 cell lines using qRT-PCR and western blot analyses. As depicted in Figures 4A, B, U251 and U87 cells exhibited significantly higher expression of ALKBH5 mRNA and protein than A172, SGH44, and LN229 cells. Accordingly, U251 and U87 cells were chosen for the subsequent in vitro experiments. We found that ALKBH5 protein expression was significantly inhibited in both cell types by siRNA targeting ALKBH5, as measured by western blot (Figure 4C). MTT assay was then used to explore the contribution of ALKBH5 expression to the proliferation of U251 and U87 cells. As predicted, downregulation of ALKBH5 significantly reduced glioma cell survival (Figure 4D). In addition, flow cytometry analysis revealed that ALKBH5 knockdown significantly increased apoptosis in U251 and U87 cells (Figure 4E). These data strongly indicate that ALKBH5 serves as an important oncogene by promoting cell growth and inhibiting cell apoptosis in vitro.

\section{miR-193a-3p Suppresses Cell Survival and Enhances Cell Apoptosis by Inhibiting ALKBH5-Regulated AKT Activation}

To determine whether ALKBH5 is a key regulator of miR-193a-3pinduced cell growth and apoptosis, U251 and U87 cells were transfected with full-length ALKBH5 to overexpress miR-193a-3p. qRT-PCR and western blot analysis revealed that miR-193a-3p mimics significantly decreased ALKBH5 mRNA and protein expression levels and this result could be partly restored by ALKBH5 treatment in glioma cells (Figures 5A, B). MTT and colony-forming assays showed that overexpression of ALKBH5 in U251 and U87 cells transfected with miR-193a-3p mimics alleviated the decreased growth observed in cells transfected with miR-193a$3 p$ mimics alone (Figures 5C, D). Flow cytometry data revealed that the upregulation of ALKBH5 could reverse the synergistic effect of miR-193a-3p overexpression on the apoptosis of glioma cells (Figure 5E). These data imply that miR-193a-3p inhibits cell survival and promotes cell apoptosis via an ALKBH5dependent mechanism. 
A

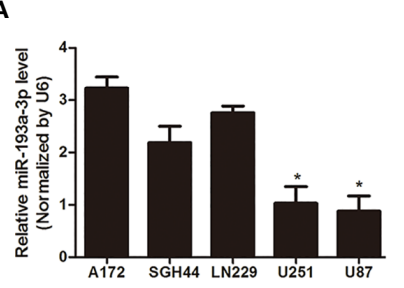

D
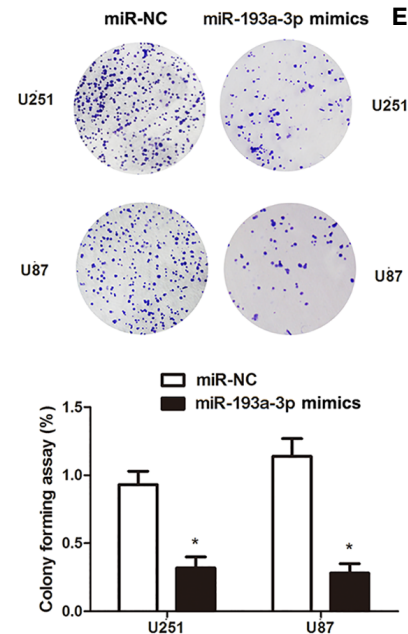

F

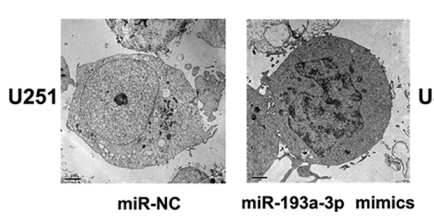

B

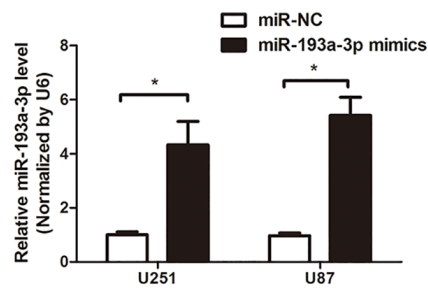

E $\quad$ miR-NC

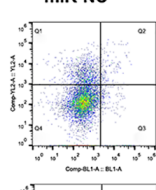

miR-193a-3p mimics

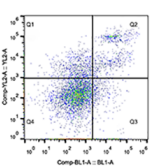

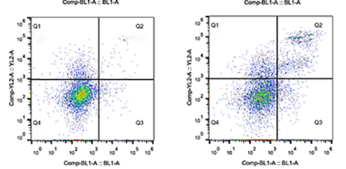

$\square$ miR-NC

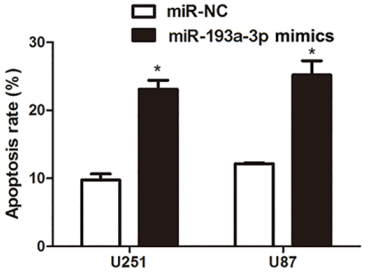

U251

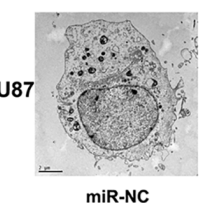

C

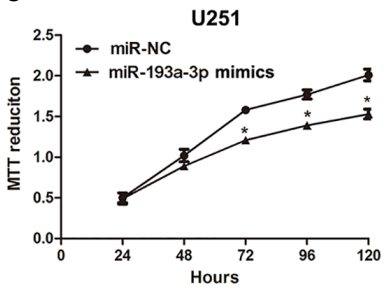

G

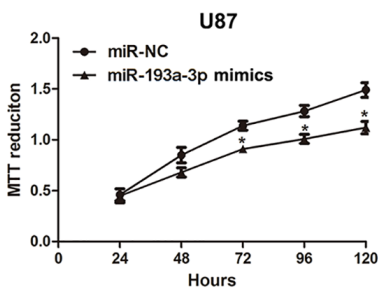

U87

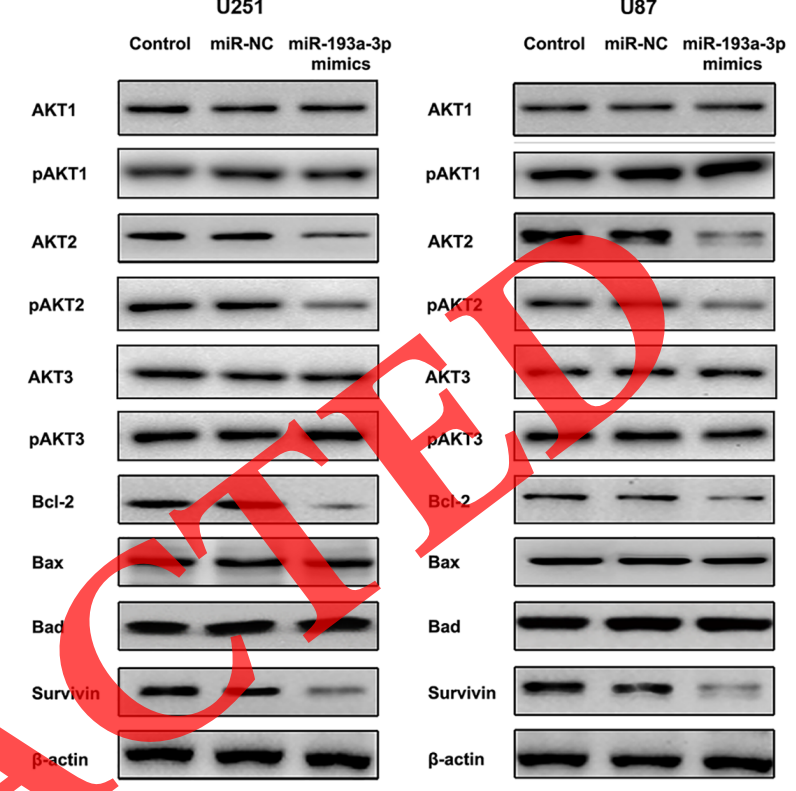

FIGURE 2 | (A) Expression levels of miR-193a-3p in glioma Cell lines (A172, SGH44, LN229, U251, and U87) were determined by qRT-PCR. *P<0.05. (B) U251 and U87 cells were transfected with miR-193a-3p mimies, followed b qRT-PCR analysis of miR-193a-3p expression. ${ }^{*} \mathrm{P}<0.05$. (C) Cell growth was determined in U251 and U87 cells transfected with miR-193a-3p mimics by MTT assay at 24, 48, 72, 96, 120h. *P<0.05. (D) Top: Effect of miR-193a-3p overexpression on U87 and U251 cell anchorage-dependent growth was determined by colony formation assay. Bottom: Quantitative analysis of anchorage-dependent growth showing a significant decrease in cell proliferation after miR $193 a-3 p$ transfection. $\mathrm{P}<0.05$. (E) Top: The number of apoptotic cells was significantly higher in U87 and U251 cells transfected with miR-193a-3p mimics as measured by Pl staining and flow cytometry. Bottom: Quantification of scatter plots showing apoptotic cells as a percent of the total cell number examined by flow cytometry. $\mathbf{P}<0.05$. (F) The miR-193a-3p-transfected cells revealed typical apoptosis features, such as nuclear fragmentation, chromatin condensation and membrahne blebbing. (G) Altered total AKT2, p-AKT2, Bcl-2 and Survivin are associated with overexpression of miR193a-3p, while no changes were observed in total AKT1, total AKT3, p-AKT1 and p-AKT3. Detection of AKT signaling molecules and apoptosis-associated proteins in U251 and U87 glioma cells by Western bl

The connection between miR-193a-3p and ALKBH5 in glioma cells prompted to investigate the activation of the AKT2 signaling pathway in response to changes in ALKBH5 expression. After a Lenti-ALKBH5-induced increase in the expression of ALKBH5 in glioma cells, ALKBH5 activated the AKT2 signaling pathway through enhancing the expression levels of total AKT2 and p-AKT2 without influencing the expression levels of total AKT1, total AKT3, p-AKT1, and pAKT3. Meanwhile, Bcl-2 and Survivin expressions were elevated in glioma cells treated with Lenti-ALKBH5 (Figure 5F). Importantly, upregulation of ALKBH5 significantly abrogated the decrease in total AKT2 and phosphorylated AKT2 expression in cells overexpressing miR-193a-3p (Figure 5F). In addition, ALKBH5 rescued the inhibitory effect of miR-193a-3p on Bcl-2 and Survivin expression in glioma cells (Figure 5F). These data indicated that miR-193a-3p-induced apoptosis in gliomas is likely related to the suppression of the AKT2 signaling pathway, which is regulated by ALKBH5.

\section{ALKBH5 Interacts With AKT2}

To explore how ALKBH5 regulates AKT2, we started by determining whether ALKBH5 physically interacts with AKT2. We co-transfected U87 cells with ALKBH5 expression plasmid and AKT2 expression plasmid. The mRNA and protein expression levels of both AKT2 and ALKBH5 were significantly upregulated after transfection by RT-qPCR and western blot analysis (Figures 6A, B). We then conducted IP to investigate their interaction. The results indicated that IP of AKT2 co-immunoprecipitated ALKBH5 (Figures 6C, D). In addition, IP of ALKBH5 also co-immunoprecipitated 
A

C

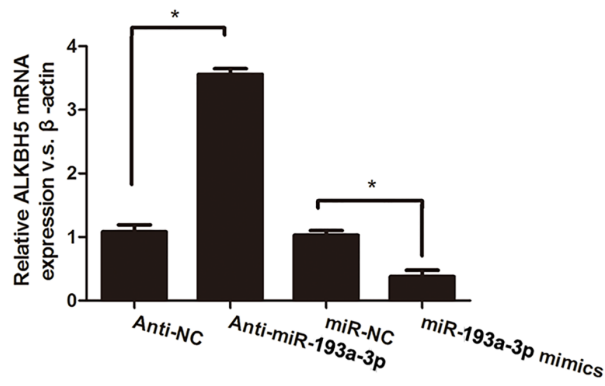

E

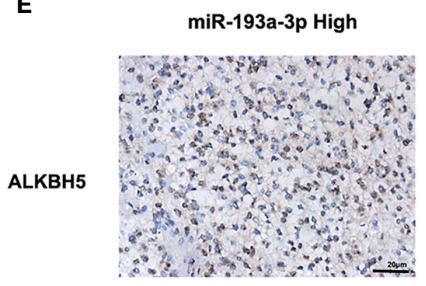

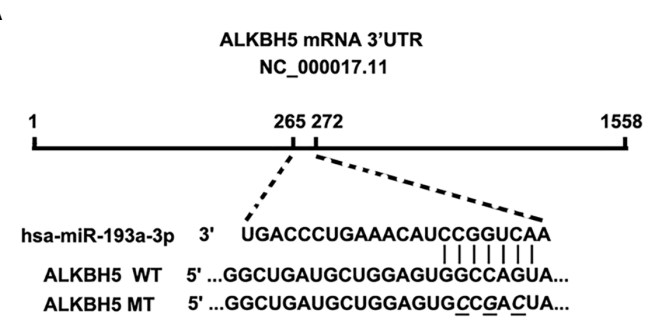

B

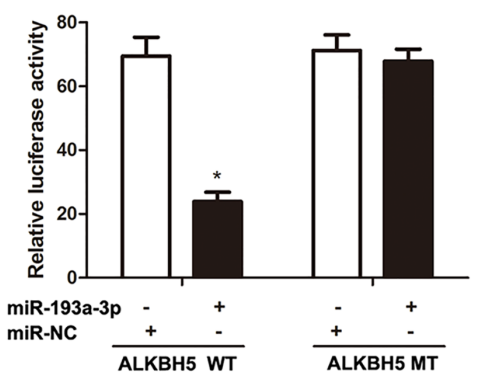

D

$$
\text { Anti-NC Anti-miR-193a-3p miR-NC miR-193a-3p mimics }
$$

ALKBH5

$\beta$-actin

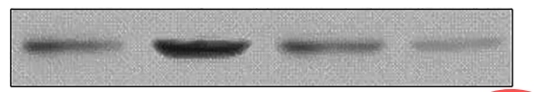

(1)
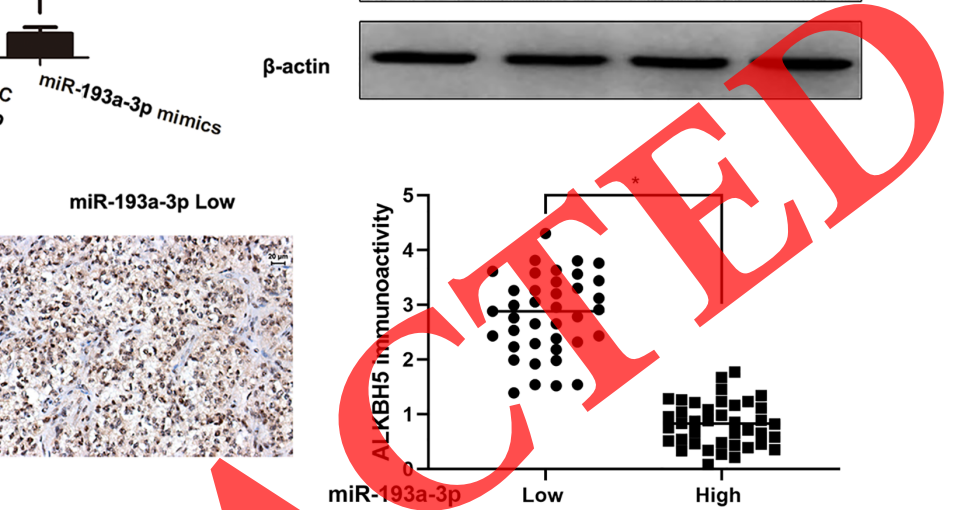

$\mathbf{F}$
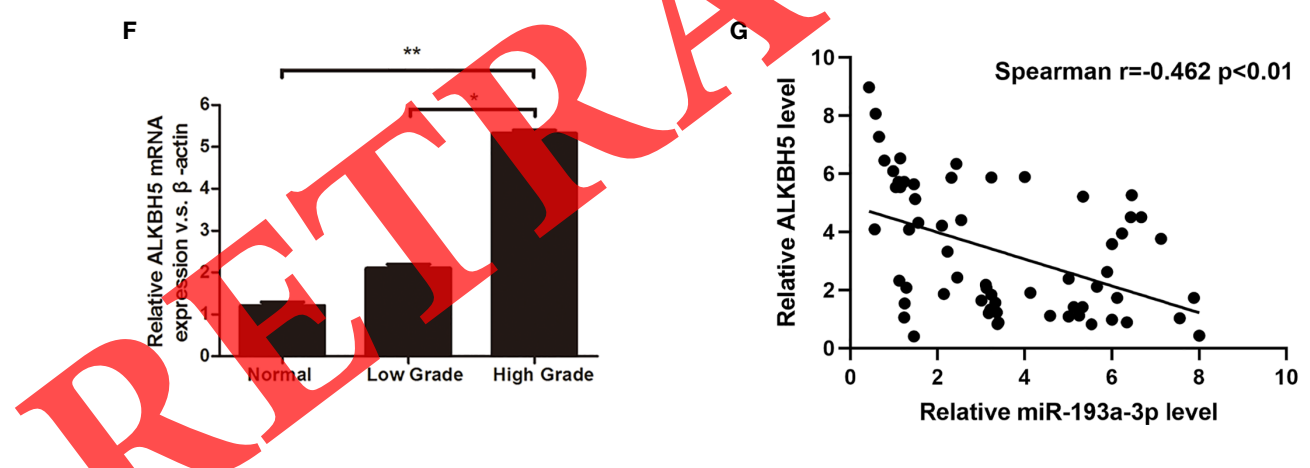

FIGURE 3 | (A) Sequence of wild type and mutant miR-193a-3p target sites in the ALKBH5. (B) The analysis of the relative luciferase activities of ALKBH5-WT, ALKBH5-MT in U87 glioma cells. $\mathrm{*}<0.05$. (C) Relative ALKBH5 mRNA levels in U87 cells transfected with miR-NC, miR-193a-3p mimics, anti-NC and anti-miR-193a-3p. ${ }^{*} \mathrm{P}<0.05$. (D) ALKBH5 protein levels were determined by Western blot in U87 cells transfected with miR-NC, miR-193a-3p mimics, anti-NC and anti-miR-193a-3p. (E) Left: Representative immunostaining showed negative expression of ALKBH5 in miR-193a-3p high-expressing glioma tissues and positive expression of ALKBH5 in miR-193a-3p low-expressing tumor. Right: Quantitative analysis of Ki-67 immunoactivity. ${ }^{*} \mathrm{P}<0.05$. (F) The expression levels of ALKBH5 mRNA in gliomas of different pathological grade were determined by GRT-PCR. ${ }^{*}<<0.05$ and ${ }^{*} P<0.01$. (G) Correlation between ALKBH5 mRNA expression and miR-193a-3p expression in human glioma tissues. $r=-0.503, P<0.01$.

AKT2 (Figure 6C). Moreover, the interaction between AKT2 and ALKBH5 was in an RNA-independent manner, because the interaction was mainly the same with and without RNase A treatment (Figures 6D, E). We further explored whether they endogenously interacted with each other by performing IP with antibodies against AKT2 and indeed found that ALKBH5 (two known isoforms indicated with arrowheads) could be coimmunoprecipitated (Figure 6F). These results imply that
ALKBH5 can physically interact with AKT2 through an RNAindependent mechanism in glioma cells.

\section{Identification of AKT2 mRNA as a Direct Target of ALKBH5 in Glioma}

To further explore the relationship between ALKBH5 and AKT2, we analyzed the effects of ALKBH5-dependent $\mathrm{m}^{6} \mathrm{~A}$ demethylation on AKT2 mRNA. Eight potential $\mathrm{m}^{6} \mathrm{~A}$ 
A

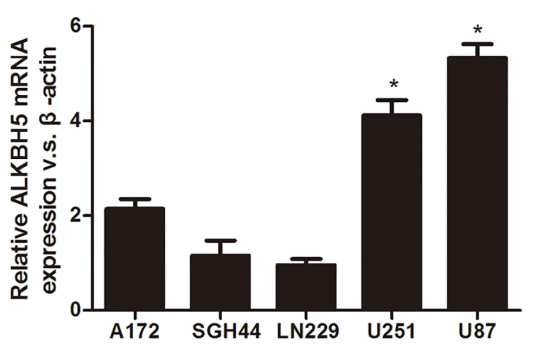

C

ALKBH5

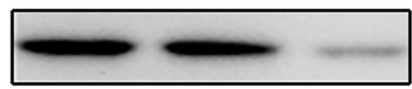

$\beta$-actin

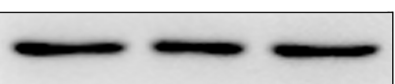

Control GFP siRNA ALKBH5 siRNA
B

$\begin{array}{llll}\text { A172 } & \text { SGH44 LN229 U251 U87 }\end{array}$

ALKBH5

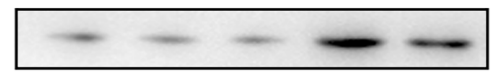

$\beta$-actin

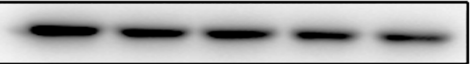

D

U251

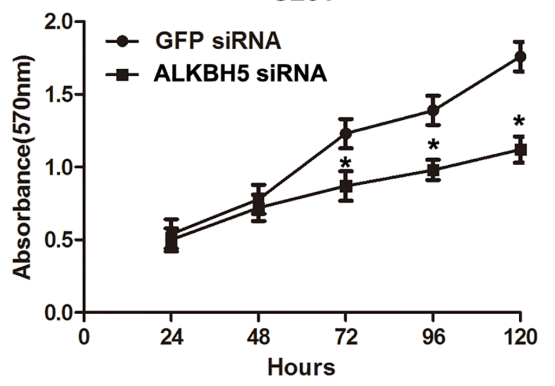

E
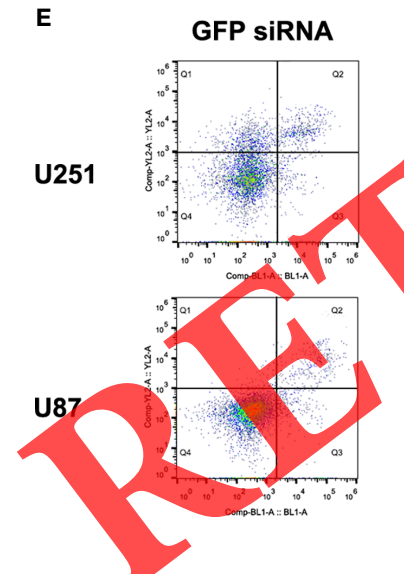

ALKBH5 SIRNA
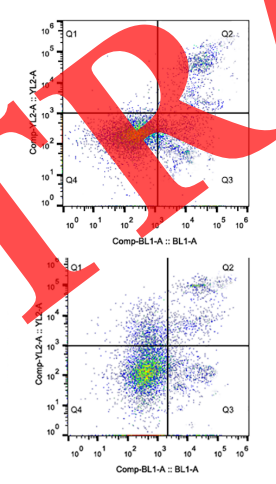

ALKBH5

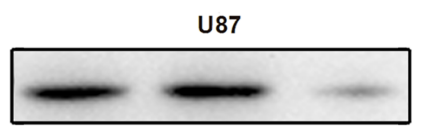

$\beta$-actin

Control GFP siRNA ALKBH5 siRNA
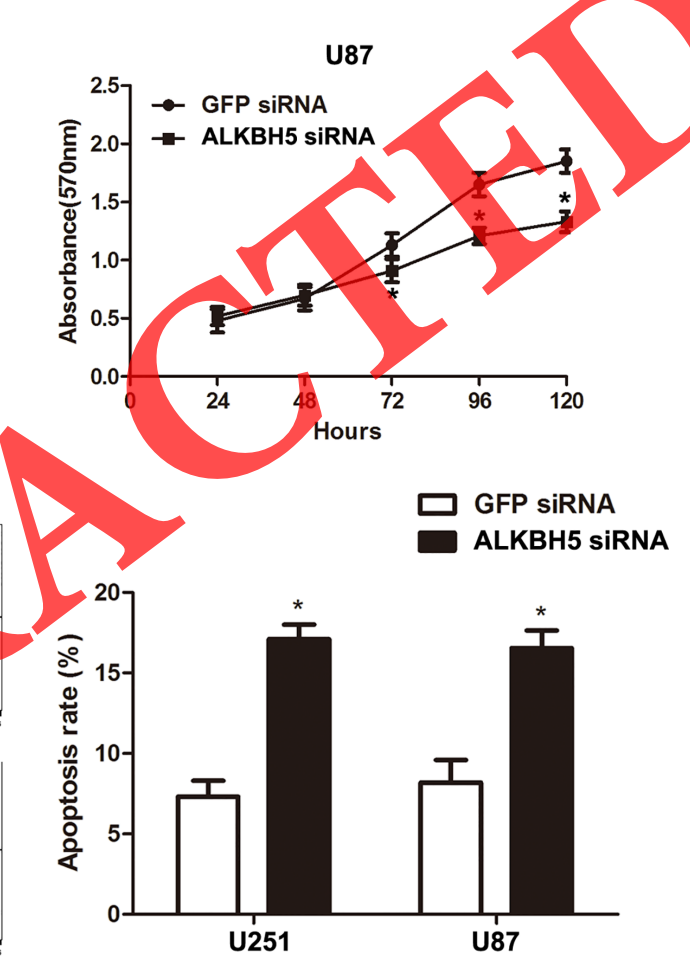

FIGURE 4 | (A) Expression mRNA levels of ALKBH5 in glioma cell lines (A172, SGH44, LN229, U251, and U87) were determined by qRT-PCR. *P<0.05. (B) Expression protein levels of ALKBH5 in glioma cell lines (A172, SGH44, LN229, U251, and U87) were determined by Western-blot analysis. (C) Expression of ALKBH5 was significantly decreased in U251 and U87 cells transfected with ALKBH5-targeted siRNA by Western blot. (D) Effect of ALKBH5 knockdown on U251 and U87 cell proliferation as measured by MTT assay at $24,48,72,96,120 \mathrm{~h}$. ${ }^{*} \mathrm{P}<0.05$. (E) The number of apoptotic cells was significantly higher in U251 and U87 cells transfected with ALKBH5-targeted siRNA as measured by flow cytometry. ${ }^{\star} \mathrm{P}<0.05$.

modification sites in AKT2 mRNA were identified by SRAMP (http://www.cuilab.cn/sramp), a m6A modification site predictor (Figure 7A). We then conducted MeRIP-qPCR to clarify whether ALKBH5 could directly mediate the $\mathrm{m}^{6} \mathrm{~A}$ methylation of AKT2 mRNA. A significant increase in the $\mathrm{m}^{6} \mathrm{~A}$ abundance of AKT2 mRNA upon suppressed ALKBH5 was observed in the m6A-RIP group, while the $\mathrm{m}^{6} \mathrm{~A}$ level did not differ in the IgGRIP group (Figure 7B). In addition, downregulation of ALKBH5 inhibited the stability of AKT2 mRNA following the treatment with actinomycin D (ActD, transcription inhibitor) in U87 glioma cells (Figure 7C). Moreover, ALKBH5 knockdown promoted the degradation of AKT2 following the treatment with cycloheximide (CHX, translation inhibitor) in U87 cells (Figure 7D). Conversely, overexpression of ALKBH5 induced a significant promotion in the AKT2-mRNA stability (Figure 7E) and a suppression in the AKT2-protein degradation (Figure 7F). 
A

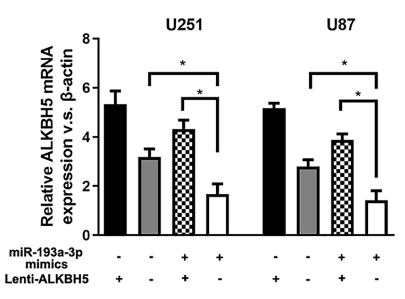

B

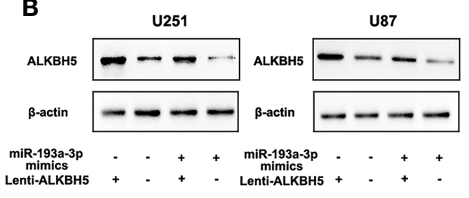

D
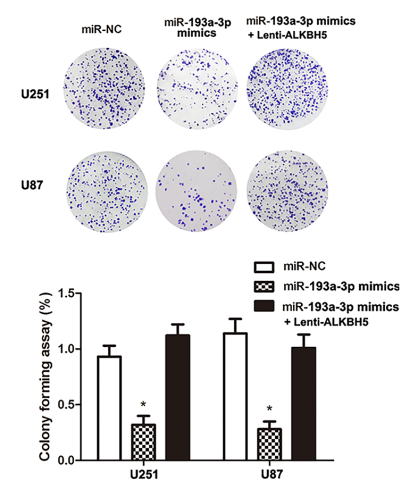

C

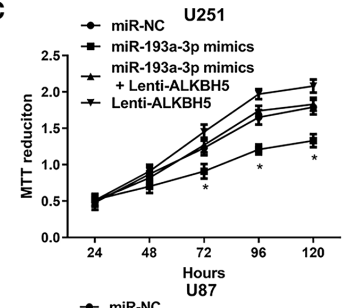

- miR-193-3p mimics

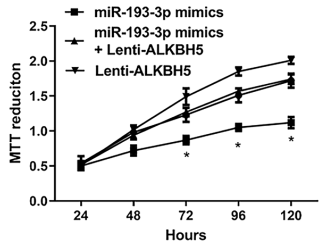

E

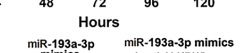

U251
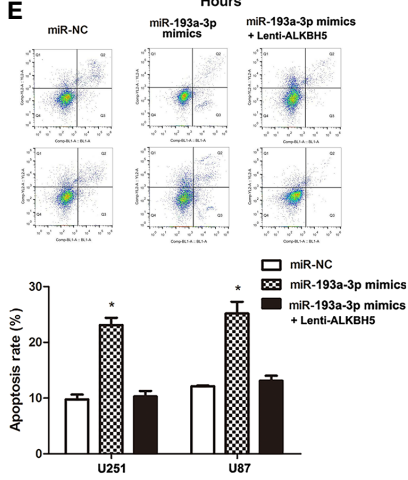
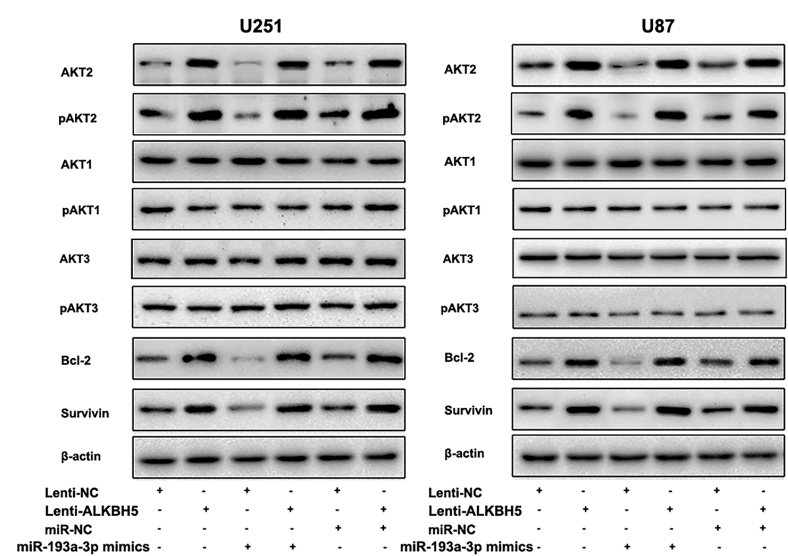

miR-193a-3p mimics

U251

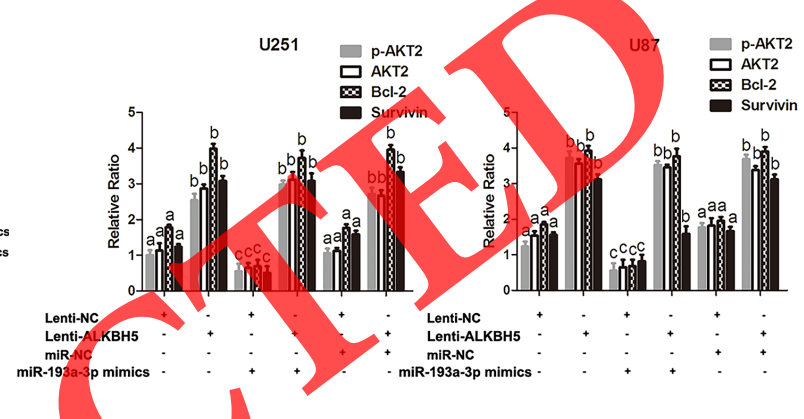

FIGURE 5 | (A) qRT-PCR analysis of glioma cells co-transfected with miR-193aco-transfected with miR-193a-3p mimics and Lenti-ALKBH5. (C) MTT assay of U251 and U87 cells co-transfected with miR-193a-3p mimics and Lenti-ALKBH5 or the control. ${ }^{*} \mathrm{P}<0.05$. (D) Colony formation assay of U251 and U87 cells co-transfected With miR-193a-3p mimics and Lenti-ALKBH5 or the control. *P<0.05. (E) Apoptosis assay of U251 and U87 cells co-transfected with miR-193a-3p mimics and Lenti-ALKBH5 or the control. *P<0.05. (F) Top: ALKBH5 overexpression blocks the effect of miR-193a-3p on total AKT2 and AKT2 phosphorylation expression without affecting total AKT1, total AKT3, p-AKT1 and p-AKT3expression. MiR-193a-3p suppresses Bcl-2 and Survivin protein expression throughALKBH5. Bottom: Quantitative analysis of Western blot analysis. Values representing the mean $\pm \mathrm{SD}(\mathrm{n}=3)$ with different letters are significa

These results suggested that ALKBH5 demethylates the $\mathrm{m}^{6} \mathrm{~A}$ modification on AKT2 mRNA and ALKBH5-regulated $\mathrm{m}^{6} \mathrm{~A}$ demethylation enhances AKT2 expression in glioma cells.

\section{miR-193a-3p Inhibits Tumor Sizes And Promotes Apoptosis In Vivo}

To further explore whether altered miR-193a-3p expression can influence the tumor growth, we established a U87 xenograft model by subcutaneously inoculating with U87 cells which were transfected with miR-193a-3p precursor sequence on nude mice. As illustrated in Figures 8A, B, upregulation of miR-193a-3p obviously reduced the size and weight of subcutaneous tumors. BrdUrd incorporation showed that miR-193a-3p overexpression resulted in a significant decrease in cell proliferation in vivo (Figure 8C). TUNEL staining indicated that tumors resulting from miR-193a-3p-mimics-transfected U87 cells revealed a significantly higher percentage of apoptotic cells in vivo (Figure 8D). To confirm that the tumor inhibition and increased apoptosis are correlated with miR-193a-3p expression, we explored the expression level of miR-193a-3p in tumors derived from U87 cells transfected with miR-193a-3p mimics. A significant overexpression of miR-193a-3p was observed in miR-193a-3p-transfected tumors (Figure 8E). Moreover, the miR-193a-3p-overexpressed U87 tumors showed a significant decrease in ALKBH5, AKT2, Bcl-2 and Survivin protein expression by Western blot analysis (Figure 8F), while there was no obvious difference in the expression level of AKT1, AKT3, Bad and Bax between miR-NC group and miR-193a-3p group (Figure S2). Token together, all these results indicate the antitumor effects of miR-193a-3p on glioma cells via ALKBH5 mediation of the AKT2-induced intrinsic apoptosis signaling pathway in vivo.

\section{DISCUSSION}

Recent studies have indicated that miR-193a-3p is decreased in cells derived from prostate, lung, breast, and colorectal tumors $(7-10,23)$, but the biological role of miR-193a-3p in human gliomas and the mechanism of miR-193a-3p-induced antitumor 


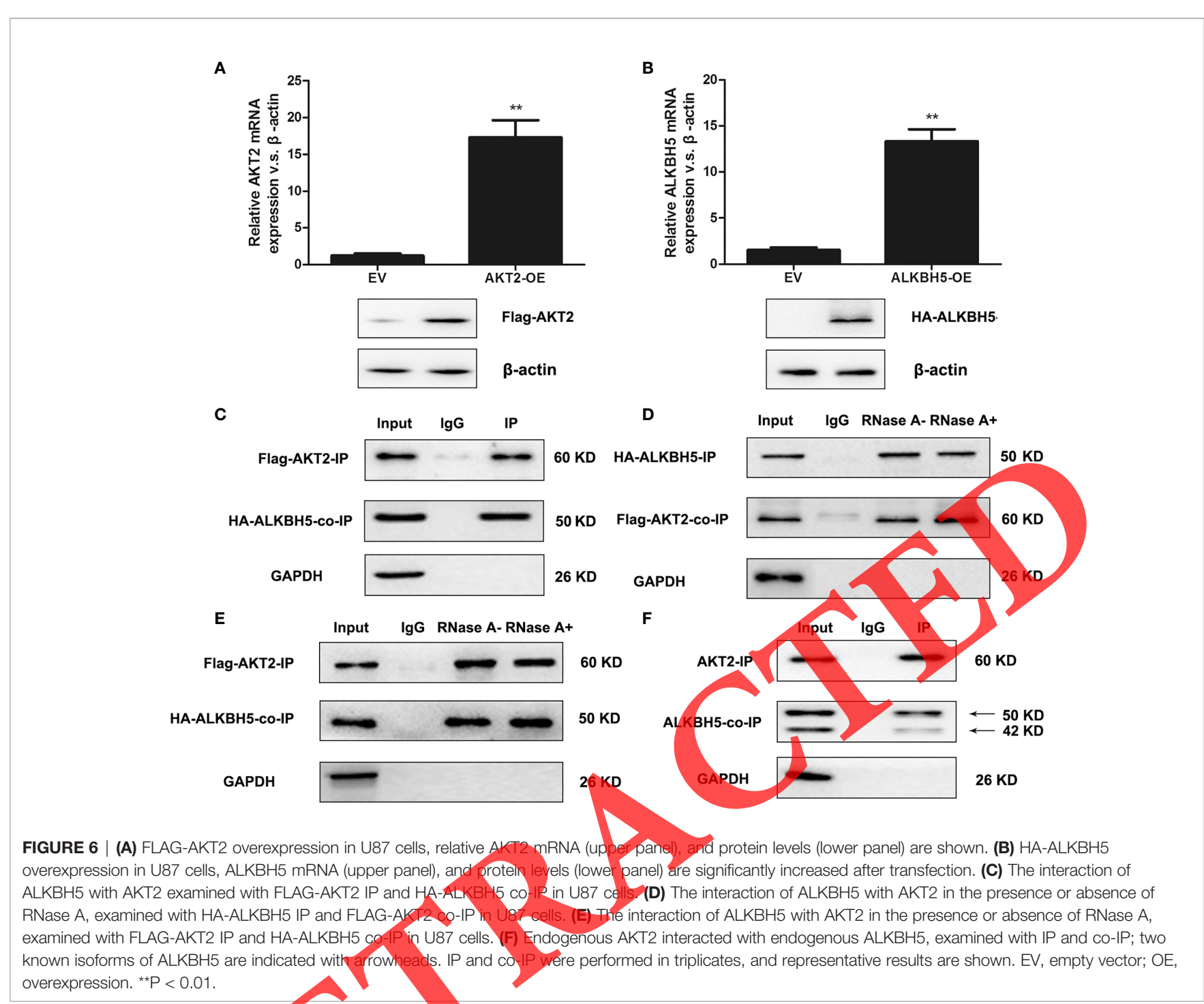

effects in tumors have not been well documented. Here, we demonstrated that miR-193a-3p is frequently underexpressed in glioblastoma tissues and U87 and 2251 malignant glioma cell lines. Interestingly, we found miR-193a-3p was overexpressed in A172 malignant glioma cells and overexpression of miR-193a-3p in A172 cells did not cause significant change in cell growth as measured by MTT assay (Figure S3), suggesting that the miR193a-3p may play a different role in A172 cells. These results are consistent with previous studies that the difference of A172, U87 and U251 malignant glioma cells at genetic level could result in different signaling pathways and tumorigenicity $(24,25)$. In addition, our results indicate that miR-193a-3p is correlated with the pathological grade, with its lowest level of expression in high-grade glioma and its highest expression in normal brain. As expected, the introduction of miR-193a-3p inhibited the proliferation and promoted the apoptosis of U251 and U87 cells. Moreover, no changes were found in miR-193b-3p expression in glioblastoma cells transfected with miR-193a-3p mimics by RT-PCR, confirming that miR-193a-3p is actually affected in our study. Thus, this is the first study to show that miR-193a-3p expression is inversely correlated with the pathological stage and serves as a tumor suppressor in gliomas.

One of the key molecular steps in the development of carcinogenesis is the suppression of apoptosis governed by pro- and antiapoptotic proteins (26). Numerous studies have shown that the imbalance between Bcl-2 family activity and the inhibitor of apoptosis (IAP) family in gliomas might be a critical factor in tumor proliferation $(27,28)$. The expression of $\mathrm{Bcl}-2$ is significantly higher in human glioblastoma and, in a PDGF-B-dependent mouse model of oligodendroglioma, Bcl-2 promoted tumor proliferation and progression to anaplastic disease $(29,30)$. In addition, Zhang et al. (31) found that higher expression of Survivin, a critical member of the IAP family, was correlated with worse overall survival in patients with glioma. Here, we demonstrated that overexpression of miR193a-3p dramatically enhanced the apoptosis of U251 and U87 
A
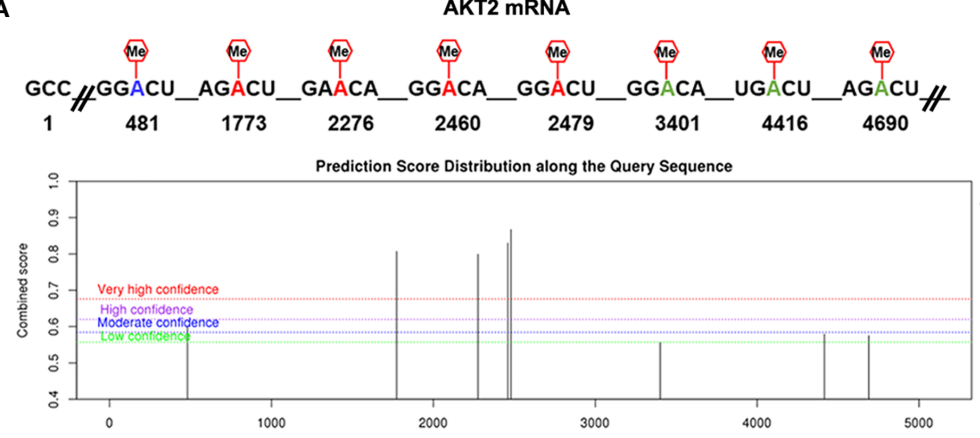

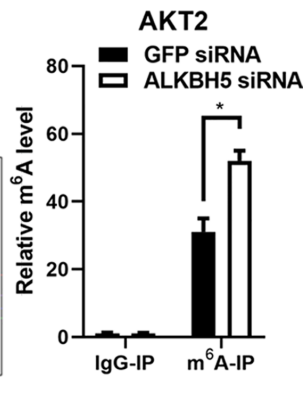

C

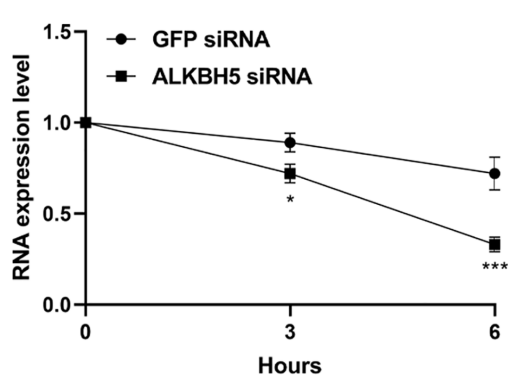

E

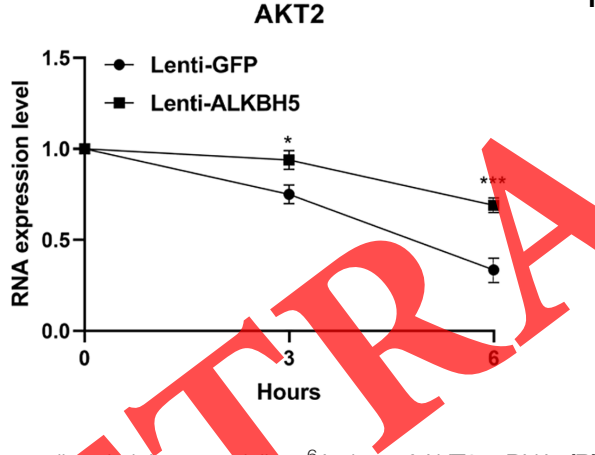

D

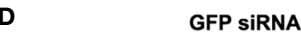

$\frac{\text { ALKBH5 SIRNA }}{\text { Oh }}$

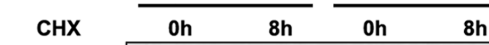

ALKBH5

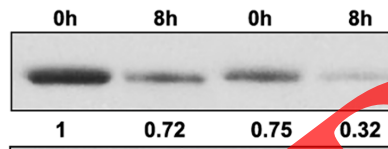

AKT2

GAPDH

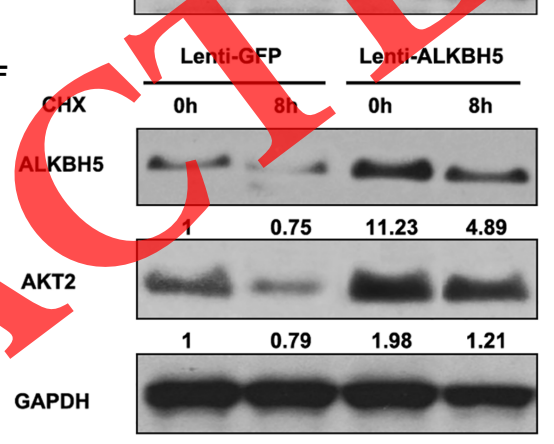

FIGURE 7 | (A) SRAMP program predictedeight potentially $m^{6} A$ sites of AKT2 mRNA. (B) Change of $m^{6} A$ modification in specific regions of AKT2 transcripts with ALKBH5 suppression in U87 cells. (C) GRT-PCR showing AKT2 transcripts stability in ActD-treated cells after transfected with ALKBH5 siRNA. (D) AKT2-protein stability after ALKBH5 inhibition. (E) QRT-PCR-showing AKT2 transcripts stability in ActD-treated cells after transfected with Lenti-ALKBH5. (F) AKT2-protein stability after upregulation of ALKBH5. ${ }^{*} \mathrm{R}<0.05 ;{ }^{* * *} \mathrm{R}<0.001$

cells compared with the miR-NC group in vivo. Furthermore, the expression levels of Bcl-2 and Survivin in glioma cells were significantly decreased after their transfection with miR-193a-3p mimics, whereas no changes were seen in the level of Bad or Bax, indicating a functional interaction between miR-193a-3p and the classic cellular intrinsic apoptosis pathway both in vitro and in vivo.

The AKT signaling pathway plays a critical role in the mediation of Bcl-2 family and IAP family proteins, which are believed to be important anti-apoptosis targets. The AKT2 protein contains an $\mathrm{SH}$-like amino-terminal region adjacent to the kinase domain, which is closely related to the v-Akt and AKT1/RAC kinases (32). The SH2-like region enables ATK2 to function as a cytoplasmic tyrosine kinase and play a critical role in the AKT signaling pathway (33). We previously examined AKT2-induced apoptosis in U87 glioma cells and found significantly lower $\mathrm{Bcl}-2$ protein expression in U87 cells underexpressing AKT2 (21). This finding is in agreement with a study by Martin et al. (34), which showed that AKT2 is an important regulator of $\mathrm{Bcl}-2$ activation via its ability to suppress calcium mobilization from the endoplasmic reticulum. These results prompted us to investigate whether miR-193a-3p could regulate the intrinsic apoptosis pathway through AKT2 alteration. We first found that overexpression of miR-193a-3p significantly suppressed AKT2 expression and activity in U251 and U87 cells without changing the expression and activity of AKT1 and AKT3. Next, we confirmed that ALKBH5 is a novel target of miR-193a-3p. Importantly, we indicated that upregulation of ALKBH5 abrogated the ability of miR-193a-3p mimics to enhance glioma cell apoptosis through overexpression of AKT2 without affecting AKT1 and AKT3 expressions or activities. Therefore, we conclude that miR-193a-3p might 
A

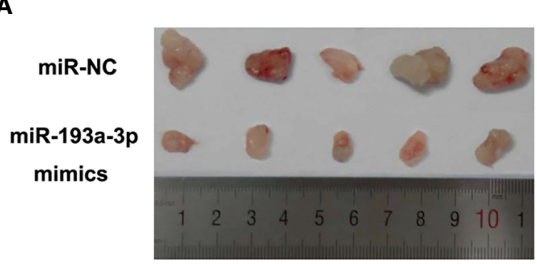

C

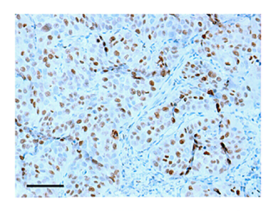

miR-NC

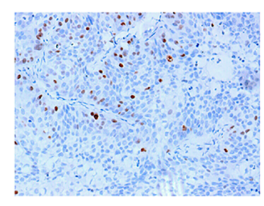

miR-193a-3p mimics
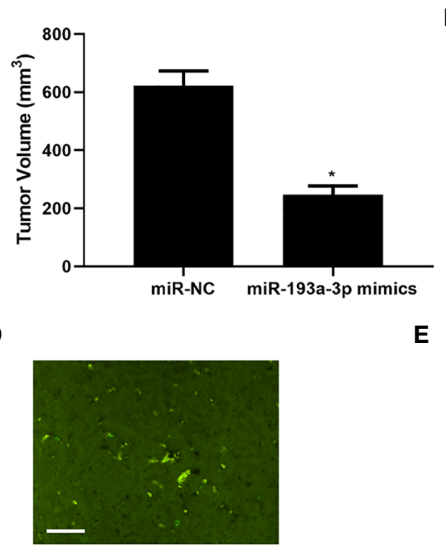

miR-NC

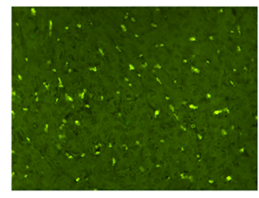

miR-193a-3p mimics

E
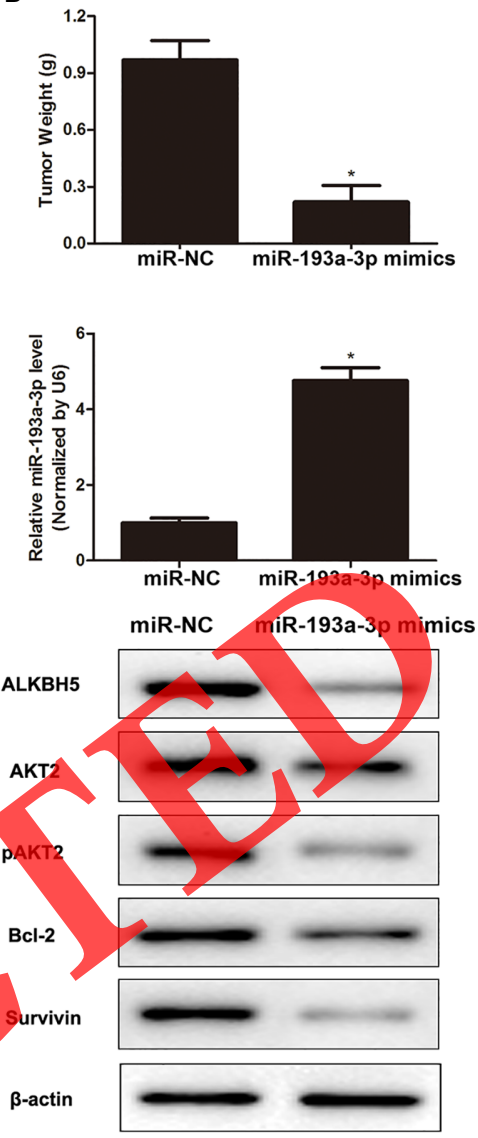

FIGURE 8 | (A) Left: Growth of implanted tumor cells was monitored by fumor volume: Right: Quantitative analysis of tumor volume. (B) Tumors were purified and weighted after the mice were sacrificed. (C) Top: Immunohistochemical analysis of BrdUrd incorporation. Bar, $20 \mu \mathrm{m}$. Bottom: Quantitative analysis of BrdUrd incorporation in U87 tumors. (D) Top: TUNEL staining in representative tumors. Bar $20 \mu \mathrm{m}$. Bottom: Quantitative analysis of TUNEL staining. (E) Expression level of miR-193a-3p in purified tumors was detected-by qRT-PCR analysis. (F) Western blot analysis was used to explore the expression of ALKBH5, AKT2, Bcl-2, and Survivin in purified tumors. ${ }^{*} \mathrm{P}<0.05$.

regulate the ALKBH5/AKT2 axis to facilitate the intrinsic apoptosis pathway of glioma cells.

Emerging evidence shows that AKT2, a human homologue of $\mathrm{v}$-akt, exerts oncogenic activity in a variety of cancers. Amplification of AKT2 was especially frequent in undifferentiated tumors, such as glioma, ovarian, breast, and prostate carcinomas $(9,33,35-37)$. Here, we introduce a new role for AKT2 activity in tumor development via physical interaction with the $\mathrm{m}^{6} \mathrm{~A}$ RNA demethylase ALKBH5. Notably, ALKBH enzymes have been demonstrated to function as nucleic acid demethylases, catalyzing the oxidative demethylation of 1methyladenine and 3-methylcytosine in DNA and RNA (38). Our study demonstrated that ALKBH5, a novel target of miR193a-3p, suppressed U251 and U87 glioma cell proliferation and promoted AKT2-induced apoptosis. Additionally, the ALKBH5AKT2 complex was found to function in U87 glioma cells through an RNA-independent mechanism. Indeed, we here revealed that loss of $\mathrm{m}^{6} \mathrm{~A}$ catalytic activity by ALKBH5 knockdown had significantly decreased $\mathrm{m}^{6} \mathrm{~A}$ enrichment of AKT2 mRNA and resulted in a suppression of AKT2 signaling pathway. Our results show that ALKBH5 increased AKT2 kinase activation and expression by a $\mathrm{m}^{6} \mathrm{~A}$ demethylase-bindinginduced mechanism, indicating that the ALKBH5/AKT2 axis play a critical role in regulating tumor process. However, how these interactions contribute to the regulation of the $\mathrm{m}^{6} \mathrm{~A}$ epitranscriptome and the functional relevance of AKT2 in the context of $\mathrm{m}^{6} \mathrm{~A}$ modification remains unclear and needs further study.

In the present study, we indicated that miR-193a-3p directly targets ALKBH5 in glioma cells. In addition, miR-193a-3p inhibits cell proliferation and promotes cell apoptosis by regulating the ALKBH5-AKT2-mediated intrinsic apoptosis signaling pathway. Moreover, we demonstrated that ALKBH5 could physically interact with AKT2 through an RNAindependent mechanism and cause a decreased $\mathrm{m}^{6} \mathrm{~A}$ enrichment of AKT2 in glioma cells. Our work adds to the 
accumulating evidence that miR-193a-3p plays a critical role in glioma tumorigenesis and that targeting of miR-193a-3p might represent an ideal gene-targeting strategy for malignant glioma treatment.

\section{DATA AVAILABILITY STATEMENT}

The raw data supporting the conclusions of this article will be made available by the authors, without undue reservation.

\section{ETHICS STATEMENT}

The studies involving human participants were reviewed and approved by the Specialty Committee on Ethics of Biomedicine Research (PJ2010-012-03) of PLA 411 Hospital. The patients/participants provided their written informed consent to participate in this study. The animal study was reviewed and approved by the Animal Care Committee of PLA 411 hospital.

\section{AUTHOR CONTRIBUTIONS}

YC and QW were responsible for designing the study and writing the manuscript. JL, CZ, LZ and $\mathrm{HC}$ were responsible for the data analysis work. CL and JQ were responsible for editing the manuscript. All authors contributed to the article and approved the submitted version.

\section{FUNDING}

This study was funded by the National Science Foundation of China (grant no. 81802489), the Shanghai Natural Science Foundation (grant nos. 18ZR1434500, 19ZR1448900 and 18411962500), and the Shanghai Jading District Health Committee Foundation (grant no.2020-KY-02).

\section{REFERENCES}

1. Lim M, Xia Y, Bettegowda C, Weller M. Current State of Immunotherapy for Glioblastoma. Nature Reviews. Clin Oncol (2018) 15:422-42. doi: 10.1038/ s41571-018-0003-5

2. Van Meir EG, Hadjipanayis CG, Norden AD, Shu HK, Wen PY, Olson JJ. Exciting New Advances in Neuro-Oncology: The Avenue to a Cure for Malignant Glioma. CA: Cancer I Clin (2010) 60:166-93. doi: 10.3322/caac.20069

3. Stegh AH, DePinho RA. Beyond Effector Caspase Inhibition: Bcl2L12 Neutralizes p53 Signaling in Glioblastoma. Cell Cycle (2011) 10:33-8. doi: $10.4161 / \mathrm{cc} .10 .1 .14365$

4. Farazi TA, Hoell JI, Morozov P, Tuschl T. MicroRNAs in Human Cancer. Adv Exp Med Biol (2013) 774:1-20. doi: 10.1007/978-94-007-5590-1_1

5. Zhang BL, Dong FL, Guo TW, Gu XH, Huang LY, Gao DS. MiRNAs Mediate GDNF-Induced Proliferation and Migration of Glioma Cells. Cell Physiol Biochem (2017) 44:1923-38. doi: 10.1159/000485883

\section{SUPPLEMENTARY MATERIAL}

The Supplementary Material for this article can be found online at: https://www.frontiersin.org/articles/10.3389/fonc.2021. 600451/full\#supplementary-material

Supplementary Figure 1 | (A) miR-193b-3p expression was measured by qRTPCR in U87 and U251 cells after miR-193a-3p mimics transfection. All expression was normalized to the level of U6 small nuclear RNA expression. Ns, no significant. (B) Authentication of biological targets of miR-193a-3p in the three databases (TargetScan, RNA22 and miRmap) through Venn diagrams software (available online: http://bioinformatics.psb.ugent.be/webtools/Venn/). Different color meant different databases.

Supplementary Figure 2 | AKT1, AKT3, Bad and Bax protein expression measured by Western-blot analysis. No significant difference was found between miR-NC group and miR-193a-3p mimics group in U87 glioma xenograft model.

Supplementary Figure 3 | Cell growth was determined in A172 cells transfected with miR-193a-3p mimics by MTT assay at 24, 48, 72, 96, $120 \mathrm{~h}$.

Supplementary Figure 4 | The original Western blots of total AKT1, total AKT2, total AKT3, p-AKT1, p-AKT2, p-AKT3, BCl-2, Bad, Bax, and Survivin in U87 and U251 cells transfected with $\mathrm{miR}-1$

Supplementary Figure 5 The original Western blots of ALKBH5 protein levels in U87 cells transfected with miR-NC, miR-193a-3p mimics, anti-NC and anti-miR-193a-3p.

Supplementary Figure $6 \mathrm{~V}$ The original Western blots of ALKBH5 expression in glioma cell hines (A172, SGH44, LN229, U251, and U87) and in U251 and U87 cells transfected with ALKBH5-targeted siRNA.

Supplementary Figure 7 | The original Western blots of ALKBH5, total AKT1, total AKT2, total-AKT3, p-AKT1 p-AKT2, p-AKT3, Bcl-2 and Survivin in U87 and 251 glioma cells co-transfected with miR-193a-3p mimics and Lenti-ALKBH5. Supplementary Figure 8 | The original images for blots of Figures 6A-F. Supplementary Figure 9 | The original Western blots of AKT2-protein stability after ALKBH5 treatment.

Supplementary Figure 10 | The original Western blots of ALKBH5, total AKT2, $\mathrm{p}-\mathrm{AKT} 2, \mathrm{Bcl}-2$, and Survivin protein expression in purified tumors.

Supplementary Table 1 | The detailed clinicopathologic characteristics of 28 patients with diffuse astrocytoma, 63 patients with glioblastomas and 32 normal brain tissues.

Supplementary Table 2 | The candidate potential target genes of miR-193a-3p in the three databases (miRmap, TargetScan and RNA22).

6. Kwon JE, Kim BY, Kwak SY, Bae IH, Han YH. Ionizing Radiation-Inducible MicroRNA miR-193a-3p Induces Apoptosis by Directly Targeting Mcl-1. Apoptosis (2013) 18:896-909. doi: 10.1007/s10495-013-0841-7

7. Fan Q, Hu X, Zhang H, Wang S, Zhang H, You C, et al. MiR-193a-3p is an Important Tumour Suppressor in Lung Cancer and Directly Targets KRAS. Cell Physiol Biochem (2017) 44:1311-24. doi: 10.1159/000485491

8. Mamoori A, Wahab R, Islam F, Lee K, Vider J, Lu CT, et al. Clinical and Biological Significance of miR-193a-3p Targeted KRAS in Colorectal Cancer Pathogenesis. Hum Pathol (2018) 71:145-56. doi: 10.1016/j.humpath.2017. 10.024

9. Tsai KW, Leung CM, Lo YH, Chen TW, Chan WC, Yu SY, et al. Arm Selection Preference of MicroRNA-193a Varies in Breast Cancer. Sci Rep (2016) 6:28176. doi: 10.1038/srep28176

10. Liu Y, Xu X, Xu X, Li S, Liang Z, Hu Z, et al. MicroRNA-193a-3p Inhibits Cell Proliferation in Prostate Cancer by Targeting Cyclin D1. Oncol Lett (2017) 14:5121-8. doi: 10.3892/ol.2017.6865 
11. Chen K, Liu MX, Mak CS, Yung MM, Leung TH, Xu D, et al. MethylationAssociated Silencing of miR-193a-3p Promotes Ovarian Cancer Aggressiveness by Targeting GRB7 and MAPK/ERK Pathways. Theranostics (2018) 8:423-36. doi: 10.7150/thno.22377

12. Garcia-Palmero I, Lopez-Larrubia P, Cerdan S, Villalobo A. Nuclear Magnetic Resonance Imaging of Tumour Growth and Neovasculature Performance in Vivo Reveals Grb7 as a Novel Antiangiogenic Target. NMR BioMed (2013) 26:1059-69. doi: 10.1002/nbm.2918

13. Schwartz S, Mumbach MR, Jovanovic M, Wang T, Maciag K, Bushkin GG, et al. Perturbation of m6A Writers Reveals Two Distinct Classes of MRNA Methylation At Internal and 5' Sites. Cell Rep (2014) 8:284-96. doi: 10.1016/ j.celrep.2014.05.048

14. Zheng G, Dahl JA, Niu Y, Fedorcsak P, Huang CM, Li CJ, et al. ALKBH5 is a Mammalian RNA Demethylase That Impacts RNA Metabolism and Mouse Fertility. Mol Cell (2013) 49:18-29. doi: 10.1016/j.molcel.2012.10.015

15. Yi YC, Chen XY, Zhang J, Zhu JS. Novel Insights Into the Interplay Between M(6)a Modification and Noncoding RNAs in Cancer. Mol Cancer (2020) 19:121. doi: 10.1186/s12943-020-01233-2

16. Grossi I, Salvi A, Abeni E, Marchina E, De Petro G. Biological Function of MicroRNA193a-3p in Health and Disease. Int J Genomics (2017) 2017:5913195. doi: 10.1155/2017/5913195

17. Gu S, Sun D, Dai H, Zhang Z. N(6)-Methyladenosine Mediates the Cellular Proliferation and Apoptosis Via MicroRNAs in Arsenite-Transformed Cells. Toxicol Lett (2018) 292:1-11. doi: 10.1016/j.toxlet.2018.04.018

18. Zhang S, Zhao BS, Zhou A, Lin K, Zheng S, Lu Z, et al. M(6)a Demethylase ALKBH5 Maintains Tumorigenicity of Glioblastoma Stem-Like Cells by Sustaining FOXM1 Expression and Cell Proliferation Program. Cancer Cell (2017) 31:591-606 e6. doi: 10.1016/j.ccell.2017.02.013

19. Xue J, Xiao P, Yu X, Zhang X. A Positive Feedback Loop Between Alkb Homolog 5 and miR-193a-3p Promotes Growth and Metastasis in Esophageal Squamous Cell Carcinoma. Hum Cell (2020) 34:502-14. doi: 10.1007/s13577-020-00458-z

20. Wang Q, Qian J, Wang J, Luo C, Chen J, Hu G, et al. Knockdown of RLIP76 Expression by RNA Interference Inhibits Invasion, Induces Cell Cycle Arrest, and Increases Chemosensitivity to the Anticancer Drug Temozolomide in Glioma Cells. J Neuro-Oncol (2013) 112:73-82. doi: 10.1007/s1 1060-013-1045-2

21. Cui Y, Lin J, Zuo J, Zhang L, Dong Y, Hu G, et al. AKT2-knockdown Suppressed Viability With Enhanced Apoptosis, and Attenuated Chemoresistance to Temozolomide of Human Glioblastoma Cells in Vitro and in Vivo. OncoTargets Ther (2015) 8:1681-90. doi: 102147OTT S83795

22. Gihr GA, Horvath-Rizea D, Hekeler E, Ganslandt O, Henkes H, Hoffmann KT, et al. Histogram Analysis of Diffusion Weighted Imaging in Low-Grade Gliomas: in Vivo Characterization of Tumor Architecture and Corresponding Neuropathology. Front Oncol (2020) 10:206 doi: 10.3389/fonc.2020.00206

23. Pu Y, Zhao F, Cai W, Meng X, Li Y, Gails. MiR-193a-3p and miR-193a-5p Suppress the Metastasis of Human Osteosarcoma Cells by Down-Regulating Rab27B and SRR, Respectively. Clin Exp Metastasis (2016) 33:359-72. doi: 10.1007/s10585-016-9783-0

24. Vogel TW, Zhuang Z, Li J, Okamoto H, Furuta M, Lee YS, et al. Proteins and Protein Pattern Differences Between Glioma Cell Lines and Glioblastoma Multiforme. Clin Cancer Res (2005) 11:3624-32. doi: 10.1158/1078-0432. CCR-04-2115

25. Agnihotri S, Burrell K, Buczkowicz P, Remke M, Golbourn B, Chornenkyy Y, et al. ATM Regulates 3-Methylpurine-DNA Glycosylase and Promotes Therapeutic Resistance to Alkylating Agents. Cancer Discovery (2014) 4:1198-213. doi: 10.1158/2159-8290.CD-14-0157

26. Mohamed MS, Bishr MK, Almutairi FM, Ali AG. Inhibitors of Apoptosis: Clinical Implications in Cancer. Apoptosis (2017) 22:1487-509. doi: 10.1007/ s10495-017-1429-4
27. Liu E, Kuang Y, He W, Xing X, Gu J. Casticin Induces Human Glioma Cell Death Through Apoptosis and Mitotic Arrest. Cell Physiol Biochem (2013) 31:805-14. doi: 10.1159/000350098

28. Ziegler DS, Keating J, Kesari S, Fast EM, Zawel L, Ramakrishna N, et al. A Small-Molecule IAP Inhibitor Overcomes Resistance to Cytotoxic Therapies in Malignant Gliomas in Vitro and in Vivo. Neuro-oncology (2011) 13:820-9. doi: 10.1093/neuonc/nor066

29. Tirapelli D, Lustosa IL, Menezes SB, Franco IM, Rodrigues AR, Peria FM, et al. High Expression of XIAP and Bcl-2 May Inhibit Programmed Cell Death in Glioblastomas. Arquivos Neuro-Psiquiatria (2017) 75:875-80. doi: 10.1590/ 0004-282x20170156

30. Doucette T, Yang Y, Zhang W, Fuller GN, Suki D, Fults DW, et al. Bcl-2 Promotes Malignant Progression in a PDGF-B-dependent Murine Model of Oligodendroglioma. Int J Cancer (2011) 129:2093-103. doi: $10.1002 / \mathrm{ijc} .25869$

31. Zhang S, Zhang C, Song Y, Zhang J, Xu J. Prognostic Role of Survivin in Patients With Glioma. Medicine (2018) 97:e0571. doi: 10.1097/MD. 0000000000010571

32. Koch CA, Anderson D, Moran MF, Ellis C, Pawson T. SH2 and SH3 Domains: Elements That Control Interactions of Cytoplasmic Signaling Proteins. Science (1991) 252:668-74. doi: 10.1126/scien

33. Cheng JQ, Godwin AK, Bellacosa A, Taguchi T, Franke TF, Hamilton TC et al. AKT2, a Putative Oncogene Eneoding a Member of a Subfamily of Protein-Serine/Threonine Kinases, is Amplified in Human Ovarian Carcinomas. Proc Natl Acad Sci USA (1992) 89:9267-71. doi: 10.1073/ pnas.89.19.9267

34. Martin VA, Wang Wh, Lipchik AM, Parker LL, He Y, Zhang S, et al. Akt2 Inhibits the Activation of NFAT in Lymphocytes by Modulating Calcium Release From Intracellular Stores. Cell Signall (2012) 24:1064-73. doi: 10.1016/j.cellsig. 2012.01.001

35. Cui Y, Wang Q, Wang J, Dong Y, Luo C, Hu G, et al. Knockdown of AKT2 Expression by RNA Interference Inhibits Proliferation, Enhances Apoptosis, and Increases Chemosensitivity to the Anticancer Drug VM-26 in U8z Glioma Cells. Brain Res (2012) 1469:1-9. doi: 10.1016/ j.brainres.2012.06.043

Liu C, Yang F. Akt2/ZEB2 May be a Biomarker for Exfoliant Cells in Ascitic Fluid in Advanced Grades of Serous Ovarian Carcinoma. Tumour Biol (2015) 36:7213-9. doi: 10.1007/s13277-015-3437-8

37. Cariaga-Martinez AE, Lopez-Ruiz P, Nombela-Blanco MP, Motino O, Gonzalez-Corpas A, Rodriguez-Ubreva J, et al. Distinct and Specific Roles of AKT1 and AKT2 in Androgen-Sensitive and Androgen-Independent Prostate Cancer Cells. Cell Signall (2013) 25:1586-97. doi: 10.1016/ j.cellsig.2013.03.019

38. Cui Q, Shi H, Ye P, Li L, Qu Q, Sun G, et al. M(6)a RNA Methylation Regulates the Self-Renewal and Tumorigenesis of Glioblastoma Stem Cells. Cell Rep (2017) 18:2622-34. doi: 10.1016/j.celrep.2017.02.059

Conflict of Interest: The authors declare that the research was conducted in the absence of any commercial or financial relationships that could be construed as a potential conflict of interest.

Copyright (๑ 2021 Cui, Wang, Lin, Zhang, Zhang, Chen, Qian and Luo. This is an open-access article distributed under the terms of the Creative Commons Attribution License (CC BY). The use, distribution or reproduction in other forums is permitted, provided the original author(s) and the copyright owner(s) are credited and that the original publication in this journal is cited, in accordance with accepted academic practice. No use, distribution or reproduction is permitted which does not comply with these terms. 